\title{
Pathway analysis identifies altered mitochondrial metabolism, neurotransmission, structural pathways and complement cascade in retina/RPE/choroid in chick model of form- deprivation myopia
}

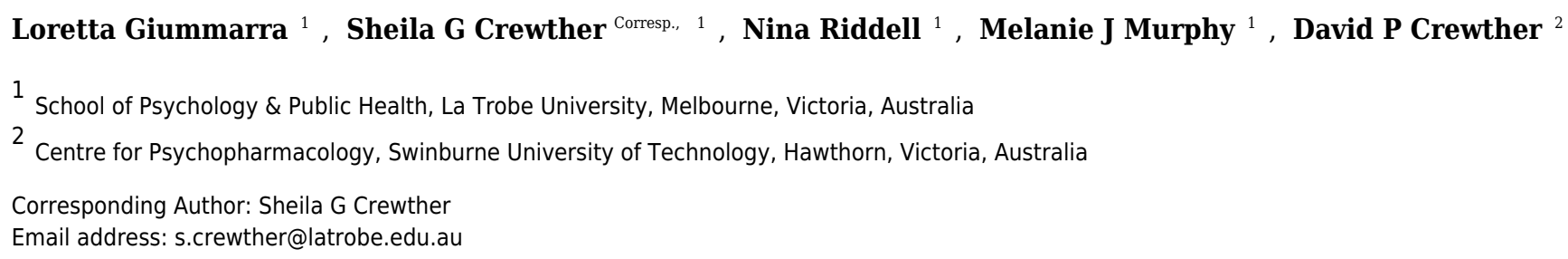

Purpose: RNA sequencing analysis has demonstrated bidirectional changes in metabolism, structural and immune pathways during early induction of defocus induced myopia. Thus, the aim of this study was to investigate whether similar gene pathways are also related to the more excessive axial growth, ultrastructural and elemental microanalytic changes seen during the induction and recovery from form-deprivation myopia (FDM) in chick and predicted by the RIDE model of myopia. Methods Archived genomic transcriptome data from the first 3 days of induction of monocularly occluded form deprived myopia (FDMI) in chicks was obtained from the GEO database (accession \# GSE6543) while data from chicks monocularly occluded for 10 days and then given up to 24 hours of normal visual recovery (FDMR) was collected. Gene set enrichment analysis (GSEA) software was used to determine enriched pathways during the induction (FDMI) and recovery (FDMR) from FD. Curated gene-sets were obtained from open access sources. Results: Clusters of significant changes in mitochondrial energy metabolism, neurotransmission, ion channel transport, G protein coupled receptor signalling, complement cascades and neuron structure and growth were identified during the 10 days of induction of profound myopia and found to correlate well with change in axial dimensions. Bile acid and bile salt metabolism pathways (cholesterol/lipid metabolism and sodium channel activation) were significantly upregulated during the first 24 hours of recovery from 10 days of FDM. Conclusions: The gene pathways altered during induction of FDM are similar to those reported in defocus induced myopia and are established indicators of oxidative stress, osmoreguatory and associated structural changes. These findings are also consistent with the choroidal thinning, axial elongation and hyperosmotic ion distribution patterns across the retina and choroid previously reported in FDM and predicted by RIDE. 
1

2

5 Loretta Giummarra $^{1}$, Sheila G. Crewther ${ }^{1 *}$, Nina Riddell ${ }^{1}$, Melanie J. Murphy ${ }^{1}$, \& David P.

6 Crewther $^{2}$

7

$8{ }^{1}$ School of Psychology \& Public Health, La Trobe University, Melbourne, 3086, Victoria,

9 Australia.

\section{Pathway Analysis identifies altered mitochondrial metabolism, neurotransmission, structural pathways and complement cascade in retina/RPE/choroid in chick model of form-deprivation myopia}

${ }^{2}$ Centre for Psychopharmacology, Swinburne University of Technology, Hawthorn, Melbourne, 3086, Victoria, Australia.

\section{*Correspondence}

Prof. Sheila Crewther

s.crewther@1atrobe.edu.au

Footnote: Reviews detailing information relating to the GSEA methodology and leading-edge analysis, include (Mootha et al. 2003; Subramanian et al. 2005) 
24 Abstract

25 Purpose: RNA sequencing analysis has demonstrated bidirectional changes in metabolism, structural and immune pathways during early induction of defocus induced myopia. Thus, the aim of this study was to investigate whether similar gene pathways are also related to the more excessive axial growth, ultrastructural and elemental microanalytic changes seen during the induction and recovery from form-deprivation myopia (FDM) in chick and predicted by the RIDE model of myopia. Methods Archived genomic transcriptome data from the first 3 days of induction of monocularly occluded form deprived myopia (FDMI) in chicks was obtained from the GEO database (accession \# GSE6543) while data from chicks monocularly occluded for 10 days and then given up to 24 hours of normal visual recovery (FDMR) was collected. Gene set enrichment analysis (GSEA) software was used to determine enriched pathways during the induction (FDMI) and recovery (FDMR) from FD. Curated gene-sets were obtained from open access sources. Results: Clusters of significant changes in mitochondrial energy metabolism, neurotransmission, ion channel transport, G protein coupled receptor signalling, complement cascades and neuron structure and growth were identified during the 10 days of induction of profound myopia and found to correlate well with change in axial dimensions. Bile acid and bile salt metabolism pathways (cholesterol/lipid metabolism and sodium channel activation) were significantly upregulated during the first 24 hours of recovery from 10 days of FDM.

Conclusions: The gene pathways altered during induction of FDM are similar to those reported in defocus induced myopia and are established indicators of oxidative stress, osmoreguatory and associated structural changes. These findings are also consistent with the choroidal thinning, axial elongation and hyperosmotic ion distribution patterns across the retina and choroid previously reported in FDM and predicted by RIDE. 
47 Myopia (short-sightedness) is the commonest visual disorder worldwide and the greatest risk

48 factor for many severe ophthalmic diseases in older individuals (Dolgin 2015). Rapidly

49 increasing prevalence has been reported among young adults in areas of South East Asia (Saw et

50 al. 2000) since the 1970s, implicating environmental influences such as changing lifestyles and

51 education as key factors in myopia development (Dolgin 2015; Junghans \& Crewther 2003;

52 Morgan et al. 2012; Schneider et al. 2010).

53

54 Many of the morphological and physiological characteristics seen in clinical myopia are

55 associated with conditions such as macular oedema, age related maculopathy (AMD), retinal

56 detachment, glaucoma and choroidal neovascularisation (CNV) (Seet et al. 2001; Yap et al.

57 1990). In particular, the elongation of the vitreal chamber, ocular volume increase, thinning of

58 the retina and choroid and reduced choroidal blood flow in clinical myopia (Borish 1949;

59 Feldman et al. 1991; Morgan et al. 2012; Moriyama et al. 2007; Yang \& Koh 2015; Zhang \&

60 Wildsoet 2015) implicate mechanisms associated with impaired transport of fluid from vitreous

61 to choroid as contributors to significantly greater physiological risk of loss of vision and

62 blindness.

63

64 Experimental models of myopia came to prominence in the late $70 \mathrm{~s}$ in monocularly occluded monkeys (Raviola \& Wiesel 1978) and chickens (Wallman et al. 1978). In animal models,

66 particularly chicken, form-deprivation (FD) is characterised by rapid ocular growth and

67 development of myopia via dramatic increases in vitreous chamber volume (Wallman et al.

68 1978), reduced choroidal blood flow and concurrent choroidal and retinal thinning (Shih et al.

69 1993a; Shih et al. 1993b; Shih et al. 1993c) (and as shown with MRI photomicrograph in Fig 1), 
70 similar to that seen in profound human myopia (Borish 1949; Feldman et al. 1991; Morgan et al.

71 2012; Moriyama et al. 2007; Yang \& Koh 2015; Zhang \& Wildsoet 2015).

72 Similarity in observations of human and animal models of myopia led to the formulation of the

73 Retinal Ion Driven Efflux (RIDE) model of myopia (Crewther 2000). This theory proposes that

74 acute blur will perturb the rate of exchange of ions and fluid between photoreceptors and the sub-

75 retinal space, concurrently affecting neurotransmission (Westbrook et al. 1999), tissue

76 osmoregulation (Crewther et al. 2006) and metabolic pathways across the posterior eye (Riddell

77 et al. 2016). As a consequence, inhibition of normal efflux of fluid across the retina/RPE would

78 result in increases in vitreous volume, axial growth and induce changes in refractive status

79 leading to myopia.

81 The relevance of the chick model in particular to understanding of human myopia has recently

82 been highlighted by Riddell \& Crewther (2017a) who firstly demonstrated that the genes near

83 human GWAS of myopia identified refractive error loci that significantly overlap with the genes

84 differentially expressed in animal transcriptome studies. Furthermore, Riddell \& Crewther

85 (2017b) also showed that the genes and proteins differentially expressed in chick myopia and

86 hyperopia models overlap significantly with those implicated in the pathogenesis of sight-

87 threatening secondary disorders.

88

89 Previous ultrastructural studies of form-deprivation in chick have demonstrated morphological

90 abnormalities in photoreceptor outer segments, retinal pigment epithelium (RPE) nuclei,

91 mitochondria and basal laminae (Beresford et al. 1998; Liang et al. 1995; Liang et al. 2004;

92 Liang et al. 1996) similar to those described elsewhere as characteristic of AMD (Datta et al. 
93 2017), CNV (Ohno-Matsui et al. 2017) and glaucoma (Kim \& Park 2017). These morphological

94 changes occur concomitantly with elemental microanalytical evidence of hyperosmotic changes

95 in ion distribution patterns across the retina, RPE and choroid (Brocker et al. 2012; Crewther et

96 al. 2006; Grubman et al. 2016; Hollborn et al. 2017; Junghans et al. 1999; Liang et al. 1995;

97 Liang et al. 2004) and reminiscent of physiologically induced hyperosmotic and oxidative stress

98 elsewhere in the brain (Brocker et al. 2012; Morland et al. 2016; Veltmann et al. 2016). Indeed,

99 oxidative stress has been suggested to contribute to the underlying mechanisms involved in

100 profound myopia pathology (Francisco et al. 2015).

101

102 Our recent RNA sequencing analysis of the genomic changes associated with early optical

103 induction of myopic and hyperopic refractive errors (Riddell et al. 2016) also suggested that

104 metabolic pathways will be altered in any genomic analysis of environmentally induced change

105 in light driven neurotransmission particularly in the most extreme of form-deprivation (FD).

106

107 Over the last decade there have been many large discovery type transcriptome studies examining

108 the genomic basis of environmentally altered eye growth in animal models of refractive error

109 development (Ashby \& Feldkaemper 2009; Brand et al. 2007; Guo et al. 2013; Guo et al. 2014;

110 He et al. 2014; McGlinn et al. 2007; Rada \& Wiechmann 2009; Riddell et al. 2016; Schippert et

111 al. 2008; Schippert et al. 2009; Shelton et al. 2008; Stone et al. 2011; Tkatchenko et al. 2006). As

112 with human genome-wide association studies (GWAS), the majority of the identified genes

113 converge into biological pathways such as cell structure, cell-cell communication,

114 neurotransmission, retinoic acid metabolism, ion transport, energy metabolism, immune system

115 and eye development (Hysi et al. 2014; Kiefer et al. 2013; Riddell \& Crewther 2017a; Stone \& 
116 Khurana 2010; Verhoeven et al. 2013). However, the large number of genes implicated in both

117 human GWAS and animal transcriptome studies have not offered a coherent explanation for the

118 anatomically derived evidence of mitochondrial and hyperosmotic stress seen across the

119 posterior retina/RPE/choroid of the FDM eye. Thus, the aim of this study was to investigate

120 whether similar gene pathways are also related to the excessive axial growth, ultrastructural and

121 elemental microanalytic changes seen during the induction and recovery from form-deprivation 122 myopia (FDM) in chick.

123

124 To test the expected association between the ultrastructural changes and the RIDE model

125 (Crewther 2000) during the induction and recovery from FDM that we have previously examined

126 (Crewther et al. 2006; Liang et al. 2004), we have reanalysed archived genomic data from

127 McGlinn et al. (2007) and a new transcriptomic dataset from chicks with FDM. The previously

128 published microarray dataset (GSE6543) (McGlinn et al., 2007) analysed retina and RPE tissue

129 at 6 and 72 hours FD myopia induction was obtained from the Gene Expression Omnibus

130 database. This dataset was reanalysed using Gene Set Enrichment Analysis (GSEA) method in

131 conjunction with our novel FDMR data using retina/RPE/choroid tissue following 10 days of

132 translucent occlusion, at time of occluder removal and then at 6 hours and 24 hours post occlude

133 removal. The RIDE model would predict that pathways associated with neurotransmission,

134 metabolism and ion solute transport would be significantly perturbed

136 Gene Set Enrichment Analysis (GSEA) (Subramanian et al. 2005) has been utilized to identify

137 key expression networks involved in induction of form-deprivation myopia (FDMI) and during

138 early recovery from form-deprivation myopia (FDMR). GSEA was originally developed to 
139 identify consistent generalized differences in the cumulative distribution in the expression of 140 genes in a biological pathway based on a priori knowledge of the gene's biological function

141 (Subramanian et al. 2005). Unlike other pathway analyses, all genes within the expression

142 dataset are considered irrespective of whether particular DEGs show statistically significant 143 differences in gene expression as identified in previous analyses.

\section{Materials and Methods}

147 FD Induction Dataset

148 Previously published microarray data from the McGlinn et al. study was obtained from the GEO

149 Database (www.ncbi.nlm.nih.gov/geo/; accession number GSE6543). The raw CEL files from

150 this study were reanalysed to complement our FD recovery profile. Although many microarray

151 studies have ascertained the transcriptome profile of refractive error development and its

152 progression (Ashby \& Feldkaemper 2009; Brand et al. 2007; McGlinn et al. 2007; Rada \&

153 Wiechmann 2009; Schippert et al. 2008; Schippert et al. 2009; Shelton et al. 2008; Stone \&

154 Khurana 2010; Tkatchenko et al. 2006), all have used optical defocus or FD in other species. The

155 McGlinn et al. study is the most comparable to ours in that similar tissue was collected ie chick

156 Retina/RPE preparation, from FD animals and analysed using the same Affymetrix microarray

157 chips. To accompany our re-analysis of this data, refraction and ocular biometrics were collected

158 from 24 chickens that were monocularly occluded at 1-week of age for $6 \mathrm{hr}(\mathrm{n}=6)$ and $72 \mathrm{hr}(\mathrm{n}=6)$

159 with an additional 12 chicks used as age-matched controls. 
160

161

162

163

164

165

166

167

168

169

170

171

172

173

174

175

176

177

178

179

180

181

\section{FD Recovery Dataset}

Animals. Twenty hatchling chicks (Leghorn $x$ New Hampshire) were utilised in this study. Fifteen chicks were form-deprived (FD) for 10 days (day 2-11 post-birth) by attaching a translucent polystyrene occluder to the periocular feathers of their right eye as previously described (Crewther et al. 2006). Separate chicks were used as aged-matched unoccluded controls $(n=5)$. Occluders were removed on day 12 and chicks were given $0 \mathrm{hr}(\mathrm{n}=5), 6 \mathrm{hr}(\mathrm{n}=5)$ or $24 \mathrm{hr}(\mathrm{n}=5)$ of normal vision to recover from form-deprivation. Chicks were raised with unlimited food and water in a controlled environment on a 12 hour light/12 hour dark cycle and with the temperature maintained at $30 \pm 0.5^{\circ} \mathrm{C}$. Illuminance was maintained at 183 lux during the $12 \mathrm{~h}$ day cycle using a $20 \mathrm{~W}$ halogen lamp. All animal work in this study was approved by the La Trobe University Animal Ethics Committee (Approval No. 05/07) and is in accordance with the Guidelines for Use of Animals in Research by the National Health and Medical Research Council (NHMRC) of Australia and the ARVO Statement for the Use of Animals in Ophthalmic and Vision Research.

Ocular Refraction, Biometric Analysis. Refractive state (dioptres (D)), vitreous chamber depth (VCD in $\mathrm{mm}$ ) and axial length (AL in $\mathrm{mm}$ ) measures were collected from all animals (induction and recovery) while animals were surgically anesthetized with an intramuscular injection of ketamine $(45 \mathrm{mg} / \mathrm{kg})$ and xylazine $(4.5 \mathrm{mg} / \mathrm{kg})$. Refraction in the experimental right eyes were determined by trained ophthalmic practitioners using retinoscopy (Keeler, Vista Diagnostic Instruments) and A-Scan ultrasonography (A-Scan III, TSL; Teknar, Inc. St Louis, USA; $7 \mathrm{MHz}$ probe) was used to measure axial dimensions. Analyses of Variance (ANOVA) was used to test group differences in refraction, AL and VCD followed by post-hoc 
182 tests if required. All dependent variables met the assumption for equal variance (Levene's Test $183 \mathrm{p}>0.05)$.

MRI imaging. MRI (4.7T) of chick FD for $72 \mathrm{~h}$ was obtained to confirm our previous

histological analysis (Liang et al. 2004) which has shown 300\% increase in choroidal thickness in the FD eye compared to fellow eye. For this, chicks were stereotaxically immobilised under surgical anaesthesia in the small bore of the magnet. Chick heart rate was also monitored. Microarray Tissue Collection and RNA Isolation. All chicks were euthanized by decapitation immediately after ocular measurements were taken. Right eyes were enucleated and the choroid/retina/RPE were taken from the posterior eye cup, placed in PrepProtect ${ }^{\mathrm{TM}}$ RNA stabilizing buffer (Miltenyi Biotec Australia Pty. Ltd., North Ryde, NSW) and stored on ice until transferred to $-20^{\circ} \mathrm{C}$ freezer. RNA was extracted using the SV total RNA isolation system (Promega Australia, NSW), including DNA digestion. The quality of the RNA samples was assessed via NanoDrop ${ }^{\circledR}$ ND-1000 Spectrophotometer and found to fall within the acceptable absorbance (260/280) range of 1.8-2.1. For Affymetrix microarrays, RNA from the right-eyes of each animal (control and experimental) was pooled in equimolar amounts by experimental condition (control $(\mathrm{n}=5), 0 \mathrm{hr}(\mathrm{n}=5), 6 \mathrm{hr}(\mathrm{n}=5)$ and $24 \mathrm{hr}(\mathrm{n}=5))$ and sent to the Australian Genome Research Facility Ltd (Walter and Eliza Hall Institute, Victoria, Australia) for microarray processing. Raw data was exported as CEL files containing probe level intensities for preprocessing with Expression Console ${ }^{\mathrm{TM}} 1.1$ (Affymetrix, Inc). This data has been submitted to GEO Database (www.ncbi.nlm.nih.gov/geo/; accession number GSE89325)

Sample pooling. Pooling of RNA was chosen as our primary aim was to identify altered biological pathways associated with experimental myopia using GSEA rather than single-gene analysis. GSEA assesses the collective changes in gene expression and identifies relevant 
205 biological pathways where these genes act (Manoli et al. 2006; Subramanian et al. 2005).

206 Pooling biological samples was originally discouraged for single gene analysis as pooling may

207 preclude variance measures in downstream statistical analysis (Peng et al. 2003). Many later

208 publications indicate that such caution is unnecessary as sample pooling does not impact

209 negatively on identifying differentially expressed genes, particularly for small experiment

210 designs and in animals within similar experimental manipulations (Bottje et al. 2012; Fu et al.

211 2011; Mengozzi et al. 2012; Mustafi et al. 2011; Zhang et al. 2007). Furthermore, such

212 limitations in sample pooling does not impact on the reliability of the GSEA algorithm which

213 requires at least a subset of genes within a pathway to be consistently ranked near the top or

214 bottom of the ordered list rather than if single gene measures were used (Manoli et al. 2006;

215 Subramanian et al. 2005). In addition, to ensure the rigor of our analyses we used a more

216 stringent statistical threshold of 0.05 for our analysis rather than the recommended 0.25 for

217 GSEA (Manoli et al. 2006; Subramanian et al. 2005).

\section{Data Pre-processing \& Normalisation}

219 To determine if the differences in chicken strain and tissues used produced confounding results, 220 we pre-processed the GSE6543 and our Affymetrix chicken chip data (CEL files) individually

221 and then together using Expression Console ${ }^{\mathrm{TM}} 1.1$ (Affymetrix, Inc). No significant outliers were

222 found in either forms of pre-processing therefore we chose to present the data that was modelled 223 together. Average background, RawQ, poly-A controls (dap, lys, phe, thr, trp) \& hybridisation 224 controls (bioD, bioC, bioD, cre) were assessed (Affymetrix 2004; Affymetrix 2006). The raw 225 data was summarised and normalised using the Robust Multichip Average (RMA) algorithm to 226 yield $\log$ base 2 expression values for each transcript. Expression values of genes with multiple

227 probe sets were then median summarised, resulting in a total of 14,298 gene measures. 
228 Expression values of the FD induction samples were averaged by condition ( $6 \mathrm{hr}$ controls $(\mathrm{n}=1)$, $2296 \mathrm{hr} F D(n=1), 72 \mathrm{hr}$ control $(\mathrm{n}=1), 72 \mathrm{hr} F D(n=1))$ to yield a single $\log 2$ expression value. This

230

231

232

233

234

235

236

237

238

239

240

241

242

243

244

245

246

247

248

249

250 was done as FDMR tissue samples were pooled prior to microarray analysis and hence resulting in one sample (ie data point) per time-point.

Although all (i.e. induction and recovery) CEL files were modelled together, average background scores ranged from 56 to 114 for the FD induction data and from 62 to 89 for the FDMR dataset. These values are mostly consistent with Affymetrix recommendations of typical average background values falling between 20 to 100 (Affymetrix 2004). Poly-A controls were all present with average signal of $d a p>t h r>p h e>l y s$. Hybridisation controls were also present with increasing signals which reflect their relative concentrations, specifically cre $>$ bioD $>$ bio $C>$ bioB . Ideally, arrays being compared should have comparable background values so these findings may have resulted from electrical noise rather than low sample quality as other parameters were consistent with manufacturer recommendations. Both GAPDH and EF1 $\alpha$ internal controls indicated that hybridisation fell within the parameters. Interestingly, the $\beta$-actin signal was above threshold for both datasets, possibly indicating that specific transcription of $\beta$-actin is altered in extreme myopia. Indeed, phototransduction requires actin filaments and microtubules to redistribute arrestin and transducin (Reidel et al. 2008) making $\beta$ actin an unreliable housekeeping gene (De Boever et al. 2008). Furthermore, our own ultrastructure studies have indicated redistribution of actin filaments in cells such as RPE and photoreceptors in form-deprivation (Liang et al. 2004). Thus, pre-processing of both datasets was rerun to mask the affymetrix housekeeping probesets for $\beta$-actin. After median summarisation, the final dataset included 14,298 genes. A report of the parameters used in the pre-processing of 
251 both the GSE6543 and our Affymetrix chicken chip datasets is included in Supplementary Table

252 1. Now available as GEO Database (www.ncbi.nlm.nih.gov/geo/; accession number GSE89325)

253

254 Gene Set Enrichment Analysis

255 The Broad Institute's Gene Set Enrichment Analysis (GSEA) software was used to determine

256 whether a priori defined sets of genes were significantly enriched (Mootha et al. 2003;

257 Subramanian et al. 2005) during the induction and recovery of FD. Curated gene sets were

258 obtained from the Molecular Signature Database (MSigDB). In particular, annotated gene sets

259 were sourced from three databases; BioCarta (http://www.biocarta.com/genes/index.asp), KEGG

260 (http://www.genome.jp/kegg/pathway.html), and Reactome (http://www.reactome.org/) and

261 chick genes were converted to human genes where possible (Supplementary Table 2). Pearson's

262 correlation was used, being the recommended metric for time-series data (Broad Institute 2012)

263 to assess changes in gene expression over the duration of occluder wear, both short-term (6hr \&

264 72hr; GSE6543) and long-term FDMI which combined data from GSE6543 (retina/RPE; 6hr \&

265 72hr) and GSE89325 (retina/RPE/choroid; 240hr). Changes in gene expression was also assessed

266 during the recovery after occluder removal (FDMR; 0hr, 6hr, \& 24hr. GSEA was also applied to

267 the control samples $(6 \mathrm{hr}, 72 \mathrm{hr}, \& 240 \mathrm{hr})$ to determine developmental or tissue-specific

268 expression profiles. Following GSEA, we performed leading-edge analysis which focuses on the

269 core gene members that account for the gene set's enrichment signal as not all members of the

270 gene-set will typically participate in a biological process underlying a disease phenotype. This

271 means that the genes that contribute most to a given pathway's enrichment (i.e. core genes) will

272 be located at the top (most upregulated) or bottom (most downregulated) of the ranked gene list

273 (Subramanian et al. 2005). 
274 To overcome gene-set redundancy and help in the interpretation of large pathway lists, clustering

275 of GSEA results was then performed using EnrichmentMap (Merico et al. 2010) for each

276 experimental group (normal development, FDMI and FDMR) and an overlap similarity

277 coefficient cut-off of 0.5 .

278

279

280

Results

281

282

283

284

285

286

287

288

289

290

291

292

293

294

295

296

\section{Ocular Biometrics for FDMI and FDMR}

We collected refractive state $(\mathrm{Rx}, \mathrm{D})$, vitreous chamber depth $(\mathrm{VCD}, \mathrm{mm})$ and axial length $(\mathrm{AL}$, $\mathrm{mm}$ ) measures for all timepoint conditions (FDMI, FDMR, control) as biometric measures were not available for McGlinn and colleagues' original microarray dataset at $6 \mathrm{hr}$ and $72 \mathrm{hr}$ of FDMI.

Thus, the earlier biometric measures presented in Fig. 2 for $6 \mathrm{hr}$ and $72 \mathrm{hr}$ induction datasets were included to provide relative indicators of growth and refraction changes expected at that number of hours of occlusion.

In response to occluder wear, AL and VCD increased and refraction became more negative with time post-occlusion under our laboratory conditions. There was a significant main effect for FD induction on refraction $[F(1,20)=33.16, p<.001]$ and time $[F(1,20)=17.99, p<.001]$. A significant interaction was also observed between $F D$ and time $[F(1,20)=10.67, p<.001]$. A significant main effect was observed for FD induction on axial length measurements $[\mathrm{F}(1$, $20)=15.40, p<.001]$ and time $[F(1,20)=33.17, p<.001]$. A significant main effect was observed for time for $\operatorname{VCD}[F(1,20)=4.31, \mathrm{p}<.05]$ but not for $\operatorname{FD}$ induction $[F(1,20)=3.61, \mathrm{p}=.07]$. 
297 For our recovery dataset (10d control, 10d FDMI, 6hr FDMR and 24hr FDMR), biometric

298 measurements and gene expression profiles were collected from the same chicks. Both refractive

299 status and axial dimensions normalised rapidly following occluder removal and $24 \mathrm{hr}$ of normal

300 visual experience (Fig 2). These measures were shown to be highly correlated ( $\mathrm{r}=.78 ;($ Fig $2 \mathrm{e})$.

301 There was a significant main effect on refraction in FD recovery $[F(1,20)=1499.27, p<.001]$ and

302 time $[\mathrm{F}(2,20)=134.0, \mathrm{p}<.001]$. A significant interaction was also observed between FD and time

$303[\mathrm{~F}(2,20)=141.46, \mathrm{p}<.001]$. Post-hoc tests revealed that refraction was significantly different at

304 all induction time-points $(\mathrm{p}<.01)$ except between 10 days of occlusion $(0 \mathrm{~h})$ and $6 \mathrm{~h}$ recovery

$305(p=.13)$. Axial length was significantly different for $F D$ recovery $F(1,20)=199.87, p<.001]$

306 and time $\mathrm{F}(2,20)=7.07, \mathrm{p}<.01]$. A significant main effect for $\mathrm{FD}$ was observed for $\operatorname{VCD}[\mathrm{F}(1$,

$30720)=146.49, \mathrm{p}<.001]$ but no main effect was seen for time $[\mathrm{F}(2,20)=2.275, \mathrm{p}=.13]$.

308

MRI of the FD chick at $72 \mathrm{hr}$ post-recovery shows substantial retinal thinning and choroidal

expansion. These observations confirm our previous histological work (Liang et al. 2004) where maximum choroidal expansion in the FD eye is approximately $300 \%$ greater than the fellow eye

[Insert Figure $1 \& 2$ here]

\section{Gene Set Enrichment Analysis}

316 To understand the underlying biological processes governing myopia development we performed pathways enrichment analysis using the Gene Set Enrichment Analysis (GSEA). We focused on identifying KEGG, Reactome, and BioCarta pathways showing expression shifts that correlated 
320 complexity of the data including sample pooling. The first analysis involved normalised log

321 expression data for short-term FD (6hr and 72hr) induction. The second dataset included

322 averaged log expression for when data was collected during the combined long-term induction

323 period (ie. $6 \mathrm{hr}, 72 \mathrm{hr}$, and $240 \mathrm{hr}$ (10d) for control and FDMI datasets) and thirdly, analysis was

324 performed on average expression values during recovery post FD occlusion (ie. 0hr (post-

325 occlusion), 6hr, and 24hr).

\section{Enrichment analysis for short-term FDM induction}

327 Our initial analysis focused on identifying KEGG, Reactome, and BioCarta pathways showing expression shifts that correlated with short-term FD to compliment previous findings by McGlinn et al. (2007). Analysis of gene expression changes between $6 \mathrm{hr}$ and $72 \mathrm{hr}$ of FD revealed 13 significantly enriched pathways. Most pathways responding to short-term FD involve mitochondrial energy metabolism (Table 1). Interestingly, none of the genes identified by McGlinn et al. (2007) were listed as a core gene for these pathways suggesting that highly regulated genes are not always driving treatment-specific biological responses. Notably, genes involved in 'One carbon pool by folate' was significantly upregulated suggesting an association between homocysteine (Hcy) and B-vitamins in short-term FD induction. Genes associated with short-term FD induction have previously been associated with AMD (Gopinath et al. 2013).

\section{Enrichment analysis for long-term FDM induction (FDMI) and recovery (FDMR)}

We next performed GSEA to identify KEGG, Reactome, and BioCarta pathways showing 
$343240 \mathrm{hr}$ (10d) for control and FDMI datasets; and 0hr (post-occlusion), 6hr, and 24hr for the

344 FDMR dataset. This analysis identified 61 significantly enriched pathways during normal

345 development, 130 during FDMI, and 1 during FDMR. We then used the Enrichment Map

346 (Merico et al. 2010) to cluster highly similar pathways into networks. Many pathways were

347 implicated during both normal (control) development and FDMI suggesting that most of these

348 pathways are likely to relate more to rate of growth rather than to the form-deprivation paradigm

349 per se. That is, given that the same pathways were identified irrespective of the experimental

350 manipulation it is likely that similar mechanisms associated with growth are likely to operate

351 under any experimental conditions. The direction of regulation i.e. up or down, would be

352 expected to be associated with rates of growth and with the age of the chicks and would be

353 predicted by the RIDE model. Summary statistics for the resulting clusters are presented in Table

354 2, and the clusters are visualized in Fig. 3 and 4.

FDMI was characterised by 19 clusters of pathways (Fig 4). Six of these clusters were also altered during normal ocular development ('cell cycle, mitotic', 'cytochrome p450', 'neurotransmission', 'phospholipid metabolism', 'signal transduction, GPCR' and 'vesiclemediated transport') but demonstrate greater signal strength during FDMI compared to normal both normal development and FDMI. 


\section{Leading edge analysis}

369 We next examined the leading-edge subsets (i.e. core genes) within the clusters identified during 370 FDMI, for potential genes that may play a role in the broader ultrastructural evidence of

371 oxidative and hyperosmotic stress previously described during FDM (Crewther et al. 2006;

372 Liang et al. 1995; Liang et al. 2004) and in brain (Brocker et al. 2012; Morland et al. 2016;

373 Veltmann et al. 2016). These clusters included mitochondrial metabolism gene sets previously

374 identified by Riddell et al. (2016) in similar aged chicks with refractive errors induced by optical 375 defocus.

377 Gene sets involved in maintaining mitochondrial metabolism (oxidative phosphorylation, TCA cycle and respiratory electron transport/ATP synthesis) or diseases involving mitochondrial

379 dysfunction (i.e Huntington's disease, Parkinson's and Alzheimer's Diseases) were significantly upregulated during short-term FDM (Table 1) and long-term FDMI (NES=2.40, p=.005; Table 2), and displayed non-significant down-regulation during the $24 \mathrm{hr}$ period of FDMR (Fig. 4). Figure 5 illustrates the relative changes in these particular pathways at different time-points. By comparison, there were no significant changes in expression of these mitochondrial metabolism pathways during normal ocular development (Table 2). This shift in metabolic regulation during induction of FDM and recovery is reminiscent of the bidirectional regulation of metabolic genes described for signed optical defocus by (Riddell et al. 2016)

Examination of the leading-edge subsets (i.e. core genes) of the mitochondrial metabolism pathways revealed 72 high scoring core genes that contributed to its significance during FDMI (Supplementary File 1b). Fourteen of these core genes are present in all mitochondrial energy 
391 metabolism gene sets and form part of mitochondrial complex I (NDUFA1, NDUFA4, 392 NDUFA5, NDUFA6, NDUFB1, NDUFB3, NDUFB6, NDUFB9, NDUFS2, NDUFS3, 393 NDUFS7), complex III (UQCR11, UQCRH) and complex IV (COX7C). Both complex I and III 394 are primary producers of reactive oxygen species (ROS) in neural tissues resulting in oxidative 395 stress when cells are unable to stabilise the ROS (Murphy 2009). Furthermore, an additional 11 396 core genes were present in 6 out of 7 gene sets with a majority coding for mitochondrial complex 397 V subunits that catalyse the conversion of adenosine diphosphate (ADP) and inorganic phosphate 398 (Pi) to ATP. Taken together, these data strongly implicate mitochondrial metabolism in the physiology of myopia.

A similar analysis of leading edge subsets relating to neurotransmission demonstrated significant 403 negative correlations with duration of occluder wear (NES=-2.42, $\mathrm{p}=0.007$; Table 2 \& Fig. 6). While these pathways showed transcriptional activation during FDMR, they did not reach the FDR cut-off of 0.05 during the short recovery period.

406

407

Five neurotransmission-related gene sets also reported significant expression shifts during 408 normal ocular development (Table 2; Fig. 6). The significance in enrichment of these pathways 409 during normal eye development was the result of 124 core genes with 10 genes common in 4 out of 5 pathways (ARHGEF9, GABRA1, GABRA2, GABRA6, GABRB2, GABRB3, GABRG2, GABRG3, GABRR1, GABRR2; Supplementary File 1a). In long-term FDMI, the signal strength

412 rose to $103 \%$ (Table 2) for neurotransmission for which there were 139 core genes ( 7 genes 413 common in 7 out of 8 pathways). The 7 genes in FDMI were serine/threonine protein kinases 
414 (BRAF, CAMK2B), ionotropic NMDA glutamate receptors (GRIN1, GRIN2A), mitogen-

415 activated protein kinase (MAPK1) and ribosomal protein S6 kinase (RPS6KA2, RPS6KA6). In

416 conjunction with a decrease in neurotransmission, analysis revealed suppression in clatherin-

417 mediated endocytosis during FDMI (NES =-2.014, $\mathrm{p}=0.032$; Table 2) suggesting that prolonged

418 FDMI results in a suppression in synaptic transmission.

419

420

421

422

423

424

425

426

427

428

429

430

431

432

433

434

435

436

[Insert Figure 6 here]

Notably, a cluster of two ion-transport related pathways showed commonalities in genes which in turn were negatively correlated with long-term occluder wear (Fig. 7). Indeed, the 'ligandgated ion channel' pathway was clustered with the neurotransmission-related pathways during normal development. The leading-edge analysis identified 15 genes that contribute to the enrichment of these pathways (ARHGEF9, GABRA1, GABRA2, GABRA5, GABRA6, GABRB2, GABRB3, GABRG2, GABRG3, GABRR1, GABRR2, GLRA1, GLRA2, GLRA3, GLRB) implicating chloride currents in the development of form-deprivation myopia (Zhang et al. 2011). This is of particular interest, as one of the aims of this study was to determine if pathways related to the mitochondrial abnormalities and ion redistribution patterns observed in our previous ultrastructural and elemental microanalysis work (Crewther et al. 2006; Liang et al. 2004; Liang et al. 1996) were also identifiable using the GSEA approach to microarray data.

[Insert Figure 7 here]

PeerJ reviewing PDF | (2018:02:24645:1:1:NEW 5 May 2018) 
437 Novel pathways associated with long-term FDMI as identified by GSEA.

438 Complement and coagulation cascades. The strongest median signal of $140 \%$ and NES of 2.71

439 during FDMI (Table 2) was found for two pathways involved in the complement and coagulation

440 cascades (Fig. 8). The Reactome 'Complement and coagulation cascade' pathway was one of the

441 two pathways within this cluster also significantly upregulated in controls (NES=2.63, $\mathrm{p}=.001$ ).

442 The core genes contributing to the upregulation of this cluster of gene sets include 49 genes, with 443 only 17 of these genes recurrent throughout this cluster. These include alpha-2-macroglobulin 444 (A2M), coagulation factors (F2, F3, F7, F8, F9, F10, F11, F13A1, F13B), fibrinogen (FGA, 445 FGG), kininogen (KNG1), serpins (SERPINC1, SERPING1), thrombomodulin (THBD) and von 446 Willebrand factor (VWF).

[Insert Figure 8 here]

448

Cytochrome 450. This cluster of gene sets produced the second highest median signal (133\%) during FDMI with an NES of 2.63 (Table 2; Fig. 9). The core genes underlying the upregulation of this cluster of pathways include the monooxygenases CYP1A2, CYP2C18, CYP3A4, and

452

453

454 455 CYP3A7 that are found in the endoplasmic reticulum (Park et al. 2014) and present in all 5 pathways within this cluster (Supplementary file $1 \mathrm{~b}$ ). The role of cytochrome p450 is in xenobiotic metabolism and subsequent synthesis of cholesterol, steroids and other lipids (Nebert \& Russell 2002). Expression of CYP1A2 is induced by the aryl hydrocarbon receptor (AHR) and HIF 1 beta (Nebert et al. 2000; Stejskalova et al. 2011). Interestingly, CYP1A1 has been reported to be involved in retinoic acid (RA) biosynthesis where manipulation of the AHR gene results in in reduced retinoic acid metabolism (Andreola et al. 1997). This is an interesting finding as CYP7A1 and CYP8B1 have also been identified as core genes in bile acid metabolism during 
460 FDMR, and are inhibited by RA (Yang et al. 2014). CYP3A4 and CYP3A7 but not CYP2C18

461 have been shown to be suppressed in the presence of inflammatory cytokines (Aitken \& Morgan

462 2007). Therefore, dysregulation of cytochrome p450 is likely to have implications for presence

463 of oxidative stress, endoplasmic reticulum stress and, if persistent, to be a serious risk factor for 464 myopia and more severe ophthalmic disease.

465

466

467

468

469

470

471

472

473

474

475

476

477

478

479

480

481

[Insert Figure 9 here]

\section{GSEA of Recovery from FDM}

GSEA to identify KEGG, Reactome, and BioCarta pathways showing expression shifts that correlated with recovery from FD by occlusion (in hours; 6hr and 240hr) was also performed. The only gene set significantly altered during the first 24 hours of refractive recovery was the bile acid and bile salt metabolism pathway. Bile acid synthesis is reliant on the interaction of peroxisome degradation and mitochondrial metabolism with the end products of cholesterol utilization being the bile acids (Lefebvre et al. 2009). Indeed, the synthesis of the bile acids is the major pathway of cholesterol catabolism in mammals. The 14 core genes involved include SLC10A1, ACOX2, AKR1D1, FABP6, CH25H, ABCC3, HSD17B4, ALB, ABCB11, CYP7B1, HSD3B7, SLCO1A2, AMACR, and CYP46A1 (Fig 10). SLC10A1 (sodium taurocholate cotransporting polypeptide), SLCO1A2 (sodium-independent Organic anion transporter), and cytochrome P450 genes, CYP7B1 and CYP46A1 are known to be involved with cholesterol pathways in brain. Other genes involved include protein members of the superfamily of ATPbinding cassette $(\mathrm{ABC})$ transporters $\mathrm{ABCC} 3$, bile salt export pump $\mathrm{ABCB} 11$, and peroxisome 
482 genes acyl-coenzyme A oxidase 2 (ACOX2), and hydroxysteroid (17-beta) dehydrogenase 4 483 (HSD17B4).

484

485

486

487

488

489

490

491

492

493

494

495

496

497

498

499

500

501

502

503

504

[Insert Figure 10 here]

Gene validation. The microarray results described here are consistent with our previously published work using RNA-seq (Riddell et al. 2016). Hence, we chose not to validate core genes from each pathway by qPCR or other molecular technique as there are $>130$ pathways identified in this study. Furthermore, validation of the core genes using qPCR is often questionable as it is reportedly subject to within-lab and technical differences (microarray vs qPCR) (Nygaard \& Hovig 2009). Microarrays have been shown to exhibit good sensitivity and specificity in detecting gene expression changes (Dago et al. 2014) and perform comparatively to RNA-seq, as indicated above in our lab particularly (Riddell et al. 2016). Additionally, single-gene analysis will not confirm significance of biological pathways identified in GSEA. For example, in the FDMI dataset, EIF4EBP1 was highly ranked at the top of the gene list for GSEA (ie. Highly upregulated; see supplementary table 3). This gene was only identified as a core gene in the 'Translation' pathway. A gene that was highly ranked at the bottom of the list (ie highly down regulated) was GDAP1. Interestingly, this gene was not listed as a core gene for any of the significant pathways identified by GSEA suggesting that highly regulated genes are not always responsible for driving treatment-specific biological responses.

\section{Discussion}

Application of the GSEA technique to the existing FDMI microarray data sets and to our new 
505 FDMR dataset demonstrate generalized statistical differences in gene pathways. Mitochondrial

506 energy metabolism was the predominant pathway upregulated in both short-term and long-term

507 FDMI. Long-term FDMI resulted in further dysregulation in several pathways including

508 suppression of neurotransmission, neuron structure/growth and subsequent ion transport

509 compared to normal development. Complement pathways were also upregulated significantly

510 over the time of form-deprivation. Furthermore, the only pathway significantly altered during the

511 first 24 hours of refractive recovery was the bile acid and bile salt metabolism pathway. This

512 pathway is reliant on the interaction of peroxisome degradation, fatty acid and mitochondrial

513 metabolism (Poirier et al. 2006) and is consistent with the downregulation of fatty acid and

514 PPAR pathways observed in chicks after 1 day of positive-lens defocus (Riddell et al. 2016).

515

516 The identification of significantly upregulated mitochondrial energy metabolism pathways is an

517 important finding. While there was a tendency towards upregulation of the mitochondrial energy

518 metabolism pathway during normal development, these pathways were consistently upregulated

519 immediately after the induction of occlusion and then gradually increased again in expression

520 during later FDMI times (Fig. 4 and Fig. 5) as refractive compensation and growth rates

521 normalized. The upregulation in mitochondrial energy pathways over the 10 days of FDMI was

522 not unexpected given previous ultrastructural evidence of abnormal photoreceptor elongation and

523 mitochondrial loss of integrity (Beresford et al. 1998; Liang et al. 1995; Liang et al. 2004), and

524 expression studies providing evidence for altered energy metabolism (Riddell et al. 2017; Riddell

525 et al. 2016) and oxidative stress (Francisco et al. 2015; Riddell \& Crewther 2017b) in myopia. 526 
527 The leading-edge genes identified as responsible for the change in these pathways primarily code

528 for the mitochondrial complexes I and III that are primary producers of ROS in the brain and are

529 associated with inability to stabilise ROS. Such instability is known to result in oxidative stress

530 (Bosch-Morell et al. 2015; Murphy 2009) which is a likely explanation for the cellular and

531 mitochondrial damage in the retina previously demonstrated ultastructurally in the FDM model

532 (Liang et al. 2004; Liang et al. 1996). Defects in mitochondrial complexes I and III as a result of

533 increased superoxide and other reactive oxygen species production (Adam-Vizi 2005) have also

534 been shown to lead to neurodegeneration and subsequent vision loss (Yu et al. 2012). Indeed,

535 this finding is consistent with recently published evidence of increased expression of TCA cycle

536 and mitochondrial metabolism genes following negative-lens wear in chick (Riddell et al. 2016)

537 and disruptions to TCA cycle metabolite abundance following FDMI in guinea pig (Yang et al.

538 2017) further cementing the importance of the mitochondrial respiratory electron transport chain

539 machinery in myopia development.

540

541 GSEA also demonstrated a greater number of suppressed transcription and signal transduction

542 pathways during FDMI (Fig 4) compared to normal development (Fig. 3). The presence of cell

543 maintenance and survival pathways identified during FDMI suggests coordinated interactions

544 between transcription factors, cell cycle components, and signalling molecules (Rue \& Martinez

545 Arias 2015), as would be expected to change in a system responding to external stimuli. The

546 majority of genes underlying the transcriptional events in FDMI were proteasome subunit genes

547 (Supplementary File 1B). The fact that these genes were suppressed during occluder wear and

548 reduced blood flow further highlights a system under severe physiological and oxidative stress as 
549 activation of the proteasome promotes cell survival against ROS-mediated oxidative stress (Choi

550 et al. 2016) as predicted by the RIDE model.

551

552 Recent evidence has also suggested a role for ROS in signal transduction by mediating a variety

553 of cellular processes (Sena \& Chandel 2012) including the regulation of neurotransmission

554 (Wilson \& Gonzalez-Billault 2015), NMDA receptor-mediated plasticity (Reviewed in Borquez

555 et al. 2016), modification of ion transport mechanisms (Cl channels and cell swelling (Liu et al.

556 2009)), endoplasmic reticulum (ER) stress and apoptosis by inhibiting WNT activation (Shen et

557 al. 2014). Indeed, when mitochondrial metabolic pathways were upregulated during FDMI,

558 NMDA-mediated signalling and ion transport pathways appeared down-regulated, implicating

559 altered glutamate and glycine signalling, water transport and chloride distribution during FDMI.

560 This NMDA signalling has previously been implicated in ocular growth control in experimental

561 myopia (Fischer et al. 1997; Fischer et al. 1998) and together are predicted by the RIDE model.

562

563 Accumulating evidence suggests co-influencing roles between oxidative stress and one-carbon

564 metabolism (1-C). In a mouse model of Parkinson's disease, high Hcy levels inhibited

565 mitochondrial complex 1 activity subsequently leading to an increase in oxidative stress and loss

566 of dopaminergic neurons in the substantia nigra (Paul et al. 2018). These findings may have

567 implications in the current understanding of the role of dopamine in myopia (Zhou et al. 2017).

568 Folate deficiency has been associated with many ocular abnormalities including ectopic lentis,

569 secondary glaucoma, optic atrophy, retinal detachment, cataracts, retinal vascular occlusive

570 disease (Ramakrishnan et al. 2006) and AMD (Gopinath et al. 2013). In astronauts, high Hyc and

571 low folate levels are associated with ophthalmic changes after space flight (Zwart et al. 2012). 
572 The microgravity-fluid shifts experienced by astronauts during space flights result in greater

573 choroidal expansion, refractive changes and abrupt increases in IOP (Lee et al. 2016) similar to

574 that observed in the chick model of FDM (Liang et al. 2004) further supporting the RIDE model

575 (Crewther 2000; Crewther et al. 2006). It is not yet known whether treatment with folic acid will

576 reverse ocular abnormalities where Hcy is elevated however Hcy levels may be an early

577 indicator of myopia (Yap \& Naughten 1998).

578

579 The identification of the complement and coagulation cascade as having highest signal strength

580 in comparison to other clusters during prolonged occlusion and induction of FDM implicates

581 previously described physiological stress mechanisms associated with constriction of the choroid

582 and reduced blood flow (Shih et al. 1993c). This is an important result as the relationship

583 between complement factors and myopia has only been reported once in humans (Long et al.

584 2013) and in the cells from posterior sclera of experimentally-induced myopia in guinea pigs

585 (Gao et al. 2015). More recently, a meta-analysis has suggested a role for the complement

586 system in experimental myopia (Riddell \& Crewther 2017b). Notably one of the core genes

587 identified in this pathway was serpin peptidase G (C1 inhibitor; SERPING1), which is reported

588 to function to maintain blood vessel integrity by binding to F12a (not identified in this study) and

589 inhibiting Bradykinin (Davis et al. 1986), a protein that promotes inflammation by increasing the

590 permeability of blood vessel walls (Greenwood 1991) and calcium-dependent release of

591 glutamate from astrocytes (Parpura et al. 1994). Interestingly, Bradykinin Receptor B2

592 (BDKRB2) is also a highly frequent core gene identified in the complement cluster and in the

593 GPCR cluster. Taken together, the regulation of SERPING1 and BDKRB2 may explain the

594 presence of edema and structural changes in the FDMI eye, possibly resulting from the increase 
595 in blood vessel permeability during constriction of the choroid via BDKRB2. BDKRB2 has

596 frequently been associated with brain edema, fluid leakage, signaling by GPCR and regulation of

597 actin cytoskeleton and potentially may allow fluids to leak into the retina/vitreous as seen in

598 pathological myopia (Bosch-Morell et al. 2015). Deficiencies in SERPING1 have also

599 previously been shown to occur in age-related macular degeneration (Ennis et al. 2008).

600

601 The identification of bile acid metabolism as the only statistically significant change during

602 FDMI was not predicted but is compatible with all omics results implicating energy, metabolic

603 and ion transport (Hysi et al. 2014; Kiefer et al. 2013; Riddell \& Crewther 2017a; Stone \&

604 Khurana 2010; Verhoeven et al. 2013) and ultrastructural fluid movements (Hysi et al., 2014;

605 Kiefer et al., 2013; Riddell \& Crewther, 2017a; Stone \& Khurana, 2010; Verhoeven et al., 2013.

606 The finding may be due to the acute time points selected for analysis given that previous

607 ultrastructural descriptions have demonstrated that minimal change in choroidal thickness occurs

608 prior to $72 \mathrm{~h}$ after occluder removal (Liang et al. 2004; Liang et al. 1996). This finding is not

609 inconsistent with our conceptualisation of FDMR in an eye that has been under physiological

610 stress for 10 days. Recent reports (Lefebvre et al. 2009; Staels \& Fonseca 2009) also indicate that

611 bile acids regulate not only their own synthesis, but also triglyceride, cholesterol, glucose, and

612 energy homeostasis and play a role in osmoregulation. Bile acid synthesis has also been shown to

613 be inhibited by all-trans retinoic acid by down regulating key bile acid synthesis and metabolism

614 enzymes, such as cytochrome oxidase (CYP7A1, CYP8B1), ion transport (SLC27A5 and

615 AKRLD1) (Mamoon et al. 2014; Yang et al. 2014) and closely associated with clock genes,

616 metabolism and epigenetic regulators (Feng \& Lazar 2012).

617 
618 The two main limitations of this study are the use of the two types of tissue (retina/RPE and

619 retina/RPE/choroid) and as discussed, the consideration of variance in gene expression between

620 the GSE6543 dataset and GSE89325 dataset due to sample pooling. Our lab has previously

621 assessed the impact of using a combination of ocular tissue in large scale genomic and proteomic

622 studies (Riddell \& Crewther 2017a) and found that regardless of the varying combinations of

623 tissues used in studies of myopia, both FD and optical defocus, similar biological mechanisms

624 were identified. Such similarity in identified biological mechanisms suggests that responses to

625 environmental manipulation that reduces focused visual information is to elicit perturbation of

626 the growth response by the whole eye across multiple tissue layers though originating in the

627 photoreceptor layer and regardless of tissue properties and functions. However, tissue type is not

628 a factor for GSEA as the analysis aims to assess combined changes in expression of genes within

629 biological networks. Furthermore, gene analysis of separate ocular tissue compared to combined

630 tissues show differing expression patterns may confer misleading results. For example, the gene

631 BMP2 that Zhang et al. (2012) reported as mainly localised in the retina of chick, has previously

632 been identified as a potential risk factor for myopia in chick retina/RPE (McGlinn et al. 2007)

633 and in chick RPE (Zhang et al. 2012). In a further cohort by the same lab (Zhang et al. 2016),

634 BMP2 was reported as non-significantly expressed in chick retina. Such contradictory results

635 raise issues about the independence of differential gene signalling by BMP2 suggesting that

636 BMP2 perturbation may be more related to other genes responding to the visual manipulation. In

637 fact, our GSEA analysis that assesses the collective gene expression changes in all genes within

638 all known biological pathways has identified BMP2 as a core gene for the immunological

639 cytokine-cytokine receptor interaction pathway. This suggests that BMP2 is possibly functioning

640 as a modulator for inflammation rather than influencing the growth signal (He et al. 2018) in the 
641 development of myopia. Thus we contend that pooling RNA from multiple tissues is not an

642 impediment to our GSEA based interpretation as evidenced by the robustness of our current

643 findings with many commonalities between FDMI and FDMR and between the different

644 methodologies and much previous research in human and animals.

645

646 Conclusions

647 We believe our analyses demonstrate that GSEA is a valuable tool in identifying altered

648 biological pathways in the chick model of refractive error, as well as providing greater statistical

649 power in identifying biological pathways not otherwise considered to be of potential

650 significance. A major strength with GSEA is the generation of further hypotheses related to the

651 understanding that many genes within a biological pathway can contribute to the underlying

652 biology of a disease (Tripathi et al. 2013). Our findings demonstrate that gene pathway changes

653 in mitochondrial energy metabolism, neurotransmission and subsequent involvement of ion

654 homeostasis are tightly coupled to axial length and refraction changes as early as $6 \mathrm{~h}$ and $72 \mathrm{~h}$

655 after application of FD. The suppression in bile acid metabolism during early recovery from

656 profound FDM highlights the importance of maintaining energy metabolism in myopia. The

657 GSEA findings provide supporting evidence for the RIDE model as well as complementing

658 earlier biometric and ultrastructural findings showing that form-deprivation occlusion leads to

659 changes in eye volume, refraction, thinning of the retina and choroid, and morphological

660 evidence for hyperosmolarity (Brocker et al. 2012; Crewther et al. 2006; Grubman et al. 2016;

661 Hollborn et al. 2017; Junghans et al. 1999; Liang et al. 1995; Liang et al. 2004). Although a

662 combination of posterior ocular tissue have been analysed in previous transcriptome studies on

663 refractive errors (McGlinn et al. 2007; Rada \& Wiechmann 2009; Riddell et al. 2016; Shelton et 
664 al. 2008; Stone et al. 2011), commonalities in differentially expressed genes have now been 665 identified regardless of species, tissue analysed and genomic platform (Riddell \& Crewther 666 2017a). Further studies using the GSEA approach may benefit from data collected from next667 generation sequencing technologies, as transcripts are sequenced for analysis and genomic 668 annotations are updated. Follow-up studies may also consider refining this analysis in specific 669 retinal cell-types/tissues including the role of the vitreous in ocular development. This study 670 provides an evidence base for further understanding of the biochemical and genetic mechanisms 671 underlying and governing environmentally induced refractive error development in chick with 672 implications for clinical myopia. However, there is need for greater understanding of the effects 673 of FD recovery over a longer period of time, particularly after $72 \mathrm{~h}$ where greatest choroidal 674 expansion has been reported (Fig 1). Future strategies to modify/supplement abnormal 675 mitochondrial dynamics and reduce ionic induction of innate immune responses, may be an 676 attractive therapeutic intervention target.

677

678

679 Acknowledgements

680 The Magnetic Resonance Image was courtesy of Egan, G., at the Howard Florey Neuroscience 681 Institute. 
682

683

684

685

686

687

688

689

690

691

692

693

694

695

696

697

698

699

700

701

702

703

704

705

706

707

708

709

710

711

712

713

714

715

716

717

718

719

720

721

722

723

\section{References}

Adam-Vizi V. 2005. Production of reactive oxygen species in brain mitochondria: contribution by electron transport chain and non-electron transport chain sources. Antioxid Redox Signal 7:1140-1149. 10.1089/ars.2005.7.1140

Affymetrix. 2004. GeneChip ${ }^{\circledR}$ Expression Analysis Technical Manual: Data Analysis Fundamentals.

Affymetrix. 2006. Affymetrix Expression Console ${ }^{\mathrm{TM}}$ Software Version 1.0 - User Guide.

Aitken AE, and Morgan ET. 2007. Gene-specific effects of inflammatory cytokines on cytochrome P450 2C, 2B6 and 3A4 mRNA levels in human hepatocytes. Drug Metab Dispos 35:1687-1693. 10.1124/dmd.107.015511

Andreola F, Fernandez-Salguero PM, Chiantore MV, Petkovich MP, Gonzalez FJ, and De Luca LM. 1997. Aryl hydrocarbon receptor knockout mice (AHR-/-) exhibit liver retinoid accumulation and reduced retinoic acid metabolism. Cancer Res 57:2835-2838.

Ashby RS, and Feldkaemper MP. 2009. Gene expression within the amacrine cell layer of chicks after myopic and hyperopic defocus. Invest Ophthalmol Vis Sci 51:3726-3735. 10.1167/iovs.09-4615

Beresford JA, Crewther SG, and Crewther DP. 1998. Anatomical correlates of experimentally induced myopia. Aust N Z J Ophthalmol 26 Suppl 1:S84-87.

Borish IM. 1949. Clinical refraction. Chicago,: Professional Press.

Borquez DA, Urrutia PJ, Wilson C, van Zundert B, Nunez MT, and Gonzalez-Billault C. 2016. Dissecting the role of redox signaling in neuronal development. $J$ Neurochem. 10.1111/jnc. 13581

Bosch-Morell F, Mérida S, and Navea A. 2015. Oxidative Stress in Myopia. Oxidative Medicine and Cellular Longevity 2015. 10.1155/2015/750637

Bottje WG, Kong BW, Song JJ, Lee JY, Hargis BM, Lassiter K, Wing T, and Hardiman J. 2012. Gene expression in breast muscle associated with feed efficiency in a single male broiler line using a chicken $44 \mathrm{~K}$ microarray. II. Differentially expressed focus genes. Poult Sci 91:2576-2587. 10.3382/ps.2012-02204

Brand C, Schaeffel F, and Feldkaemper MP. 2007. A microarray analysis of retinal transcripts that are controlled by image contrast in mice. Mol Vis 13:920-932.

Broad Institute MIoTM. 2012. Gene Set Enrichment Analysis (GSEA) User Guide. Available at http://software.broadinstitute.org/gsea/doc/GSEAUserGuideFrame.html.

Brocker C, Thompson DC, and Vasiliou V. 2012. The role of hyperosmotic stress in inflammation and disease. Biomol Concepts 3:345-364. 10.1515/bmc-2012-0001

Choi WH, de Poot SA, Lee JH, Kim JH, Han DH, Kim YK, Finley D, and Lee MJ. 2016. Opengate mutants of the mammalian proteasome show enhanced ubiquitin-conjugate degradation. Nat Commun 7:10963. 10.1038/ncomms 10963

Crewther DP. 2000. The role of photoreceptors in the control of refractive state. Progress in Retinal and Eye Research 19:421-457.

Crewther SG, Liang H, Junghans BM, and Crewther DP. 2006. Ionic control of ocular growth and refractive change. Proceedings of the National Academy of Sciences of the United States of America 103:15663-15668. 
724

725

726

727

728

729

730

731

732

733

734

735

736

737

738

739

740

741

742

743

744

745

746

747

748

749

750

751

752

753

754

755

756

757

758

759

760

761

762

763

764

765

766

767

768

Dago D, Malerba G, Ferarrini A, and Delledonne M. 2014. Evaluation of microarray sensitivity and specificity in gene expression differential analysis by RNA-seq and quantitative RTPCR. Journal of Multidisciplinary Scientific Research 20142:5-9.

Datta S, Cano M, Ebrahimi K, Wang L, and Handa JT. 2017. The impact of oxidative stress and inflammation on RPE degeneration in non-neovascular AMD. Prog Retin Eye Res 60:201-218. 10.1016/j.preteyeres.2017.03.002

Davis AE, 3rd, Whitehead AS, Harrison RA, Dauphinais A, Bruns GA, Cicardi M, and Rosen FS. 1986. Human inhibitor of the first component of complement, C1: characterization of cDNA clones and localization of the gene to chromosome 11. Proc Natl Acad Sci US A $83: 3161-3165$.

De Boever S, Vangestel C, De Backer P, Croubels S, and Sys SU. 2008. Identification and validation of housekeeping genes as internal control for gene expression in an intravenous LPS inflammation model in chickens. Vet Immunol Immunopathol 122:312317. 10.1016/j.vetimm.2007.12.002

Dolgin E. 2015. The myopia boom. Nature 519:276-278. 10.1038/519276a

Ennis S, Jomary C, Mullins R, Cree A, Chen X, Macleod A, Jones S, Collins A, Stone E, and Lotery A. 2008. Association between the SERPING1 gene and age-related macular degeneration: a two-stage case-control study. Lancet 372:1828-1834. 10.1016/S01406736(08)61348-3

Feldman EL, Randolph AE, Johnston GC, DelMonte MA, and Greene DA. 1991. Receptorcoupled phosphoinositide hydrolysis in human retinal pigment epithelium. Journal of Neurochemistry 56:2094-2100.

Feng D, and Lazar MA. 2012. Clocks, metabolism, and the epigenome. Mol Cell 47:158-167. 10.1016/j.molcel.2012.06.026

Fischer AJ, Seltner RL, and Stell WK. 1997. N-methyl-D-aspartate-induced excitotoxicity causes myopia in hatched chicks. Can J Ophthalmol 32:373-377.

Fischer AJ, Seltner RL, and Stell WK. 1998. Opiate and N-methyl-D-aspartate receptors in formdeprivation myopia. Vis Neurosci 15:1089-1096.

Francisco BM, Salvador M, and Amparo N. 2015. Oxidative stress in myopia. Oxid Med Cell Longev 2015:750637. 10.1155/2015/750637

$\mathrm{Fu} \mathrm{Y,} \mathrm{Yi} \mathrm{Z,} \mathrm{Wu} \mathrm{X,} \mathrm{Li} \mathrm{J,} \mathrm{and} \mathrm{Xu} \mathrm{F.} \mathrm{2011.} \mathrm{Circulating} \mathrm{microRNAs} \mathrm{in} \mathrm{patients} \mathrm{with} \mathrm{active}$ pulmonary tuberculosis. J Clin Microbiol 49:4246-4251. 10.1128/JCM.05459-11

Gao TT, Long Q, and Yang X. 2015. Complement factors C1q, C3 and C5b-9 in the posterior sclera of guinea pigs with negative lens-defocused myopia. Int J Ophthalmol 8:675-680. 10.3980/j.issn.2222-3959.2015.04.06

Gopinath B, Flood VM, Rochtchina E, Wang JJ, and Mitchell P. 2013. Homocysteine, folate, vitamin B-12, and 10-y incidence of age-related macular degeneration. Am J Clin Nutr 98:129-135. 10.3945/ajcn.112.057091

Greenwood J. 1991. Mechanisms of blood-brain barrier breakdown. Neuroradiology 33:95-100.

Grubman A, Guennel P, Vessey KA, Jones MW, James SA, de Jonge MD, White AR, and Fletcher EL. 2016. X-ray fluorescence microscopic measurement of elemental distribution in the mouse retina with age. Metallomics 8:1110-1121. 10.1039/c6mt00055j

Guo L, Frost MR, He L, Siegwart JT, Jr., and Norton TT. 2013. Gene expression signatures in tree shrew sclera in response to three myopiagenic conditions. Invest Ophthalmol Vis Sci 54:6806-6819. 10.1167/iovs.13-12551 
769

770

771

772

773

774

775

776

777

778

779

780

781

782

783

784

785

786

787

788

789

790

791

792

793

794

795

796

797

798

799

800

801

802

803

804

805

806

807

808

809

810

811

812

813

814
Guo L, Frost MR, Siegwart JT, Jr., and Norton TT. 2014. Scleral gene expression during recovery from myopia compared with expression during myopia development in tree shrew. Mol Vis 20:1643-1659.

He L, Frost MR, Siegwart JT, Jr., and Norton TT. 2014. Gene expression signatures in tree shrew choroid during lens-induced myopia and recovery. Exp Eye Res 123:56-71. 10.1016/j.exer.2014.04.005

He L, Frost MR, Siegwart JT, Jr., and Norton TT. 2018. Altered gene expression in tree shrew retina and retinal pigment epithelium produced by short periods of minus-lens wear. Exp Eye Res 168:77-88. 10.1016/j.exer.2018.01.005

Hollborn M, Fischer S, Kuhrt H, Wiedemann P, Bringmann A, and Kohen L. 2017. Osmotic regulation of NFAT5 expression in RPE cells: The involvement of purinergic receptor signaling. Molecular Vision 23:116-130.

Hysi PG, Mahroo OA, Cumberland P, Wojciechowski R, Williams KM, Young TL, Mackey DA, Rahi JS, and Hammond CJ. 2014. Common mechanisms underlying refractive error identified in functional analysis of gene lists from genome-wide association study results in 2 European British cohorts. JAMA Ophthalmol 132:50-56.

10.1001/jamaophthalmol.2013.6022

Junghans BM, and Crewther SG. 2003. Prevalence of myopia among primary school children in eastern Sydney. Clinical \& Experimental Optometry 86:339-345. ceo865339 [pii]

Junghans BM, Wadley RB, Crewther SG, and Crewther DP. 1999. X-ray elemental analysis differentiates blood vessels and lymphatic vessels in the chick choroid. Aust NZJ Ophthalmol 27:244-246.

Kiefer AK, Tung JY, Do CB, Hinds DA, Mountain JL, Francke U, and Eriksson N. 2013. Genome-wide analysis points to roles for extracellular matrix remodeling, the visual cycle, and neuronal development in myopia. PLoS Genet 9:e1003299.

10.1371/journal.pgen.1003299

Kim KE, and Park KH. 2017. Macular imaging by optical coherence tomography in the diagnosis and management of glaucoma. Br J Ophthalmol. 10.1136/bjophthalmol-2017310869

Lee AG, Tarver WJ, Mader TH, Gibson CR, Hart SF, and Otto CA. 2016. Neuro-Ophthalmology of Space Flight. J Neuroophthalmol 36:85-91. 10.1097/WNO.0000000000000334

Lefebvre P, Cariou B, Lien F, Kuipers F, and Staels B. 2009. Role of bile acids and bile acid receptors in metabolic regulation. Physiol Rev 89:147-191. 10.1152/physrev.00010.2008

Liang H, Crewther DP, Crewther SG, and Barila AM. 1995. A role for photoreceptor outer segments in the induction of deprivation myopia. Vision Res 35:1217-1225.

Liang H, Crewther SG, Crewther DP, and Junghans BM. 2004. Structural and elemental evidence for edema in the retina, retinal pigment epithelium, and choroid during recovery from experimentally induced myopia. Investigative Ophthalmology \& Visual Science 45:2463-2474.

Liang H, Crewther SG, Crewther DP, and Pirie B. 1996. Morphology of the recovery from form deprivation myopia in the chick. Australian and New Zealand Journal of Ophthalmology 24:41-44.

Liu HT, Akita T, Shimizu T, Sabirov RZ, and Okada Y. 2009. Bradykinin-induced astrocyteneuron signalling: glutamate release is mediated by ROS-activated volume-sensitive outwardly rectifying anion channels. J Physiol 587:2197-2209. 10.1113/jphysiol.2008.165084 
815

816

817

818

819

820

821

822

823

824

825

826

827

828

829

830

831

832

833

834

835

836

837

838

839

840

841

842

843

844

845

846

847

848

849

850

851

852

853

854

855

856

857

858

Long Q, Ye J, Li Y, Wang S, and Jiang Y. 2013. C-reactive protein and complement components in patients with pathological myopia. Optom Vis Sci 90:501-506. 10.1097/OPX.0b013e31828daa6e

Mamoon A, Subauste A, Subauste MC, and Subauste J. 2014. Retinoic acid regulates several genes in bile acid and lipid metabolism via upregulation of small heterodimer partner in hepatocytes. Gene 550:165-170. 10.1016/j.gene.2014.07.017

Manoli T, Gretz N, Grone HJ, Kenzelmann M, Eils R, and Brors B. 2006. Group testing for pathway analysis improves comparability of different microarray datasets. Bioinformatics 22:2500-2506. 10.1093/bioinformatics/bt1424

McGlinn AM, Baldwin DA, Tobias JW, Budak MT, Khurana TS, and Stone RA. 2007. Formdeprivation myopia in chick induces limited changes in retinal gene expression. Invest Ophthalmol Vis Sci 48:3430-3436. 10.1167/iovs.06-1538

Mengozzi M, Cervellini I, Villa P, Erbayraktar Z, Gokmen N, Yilmaz O, Erbayraktar S, Manohasandra M, Van Hummelen P, Vandenabeele P, Chernajovsky Y, Annenkov A, and Ghezzi P. 2012. Erythropoietin-induced changes in brain gene expression reveal induction of synaptic plasticity genes in experimental stroke. Proc Natl Acad Sci U S A 109:9617-9622. 10.1073/pnas. 1200554109

Merico D, Isserlin R, Stueker O, Emili A, and Bader GD. 2010. Enrichment map: a networkbased method for gene-set enrichment visualization and interpretation. PLoS One 5:e13984. 10.1371/journal.pone.0013984

Mootha VK, Lindgren CM, Eriksson KF, Subramanian A, Sihag S, Lehar J, Puigserver P, Carlsson E, Ridderstrale M, Laurila E, Houstis N, Daly MJ, Patterson N, Mesirov JP, Golub TR, Tamayo P, Spiegelman B, Lander ES, Hirschhorn JN, Altshuler D, and Groop LC. 2003. PGC-1alpha-responsive genes involved in oxidative phosphorylation are coordinately downregulated in human diabetes. Nat Genet 34:267-273. 10.1038/ng1180

Morgan IG, Ohno-Matsui K, and Saw SM. 2012. Ophthalmology 2 Myopia. Lancet 379:17391748.

Moriyama M, Ohno-Matsui K, Futagami S, Yoshida T, Hayashi K, Shimada N, Kojima A, Tokoro T, and Mochizuki M. 2007. Morphology and long-term changes of choroidal vascular structure in highly myopic eyes with and without posterior staphyloma. Ophthalmology 114:1755-1762. 10.1016/j.ophtha.2006.11.034

Morland C, Pettersen MN, and Hassel B. 2016. Hyperosmolar sodium chloride is toxic to cultured neurons and causes reduction of glucose metabolism and ATP levels, an increase in glutamate uptake, and a reduction in cytosolic calcium. Neurotoxicology 54:34-43. 10.1016/j.neuro.2016.03.005

Murphy MP. 2009. How mitochondria produce reactive oxygen species. Biochem J 417:1-13. 10.1042/BJ20081386

Mustafi D, Kevany BM, Genoud C, Okano K, Cideciyan AV, Sumaroka A, Roman AJ, Jacobson SG, Engel A, Adams MD, and Palczewski K. 2011. Defective photoreceptor phagocytosis in a mouse model of enhanced S-cone syndrome causes progressive retinal degeneration. FASEB J 25:3157-3176. 10.1096/fj.11-186767

Nebert DW, Roe AL, Dieter MZ, Solis WA, Yang Y, and Dalton TP. 2000. Role of the aromatic hydrocarbon receptor and $[\mathrm{Ah}]$ gene battery in the oxidative stress response, cell cycle control, and apoptosis. Biochem Pharmacol 59:65-85. 
859

860

861

862

863

864

865

866

867

868

869

870

871

872

873

874

875

876

877

878

879

880

881

882

883

884

885

886

887

888

889

890

891

892

893

894

895

896

897

898

899

900

901

902

903

Nebert DW, and Russell DW. 2002. Clinical importance of the cytochromes P450. Lancet 360:1155-1162. 10.1016/S0140-6736(02)11203-7

Nygaard V, and Hovig E. 2009. Methods for quantitation of gene expression. Front Biosci (Landmark Ed) 14:552-569.

Ohno-Matsui K, Ikuno Y, Lai TYY, and Gemmy Cheung CM. 2017. Diagnosis and treatment guideline for myopic choroidal neovascularization due to pathologic myopia. Prog Retin Eye Res. 10.1016/j.preteyeres.2017.10.005

Park JW, Reed JR, Brignac-Huber LM, and Backes WL. 2014. Cytochrome P450 system proteins reside in different regions of the endoplasmic reticulum. Biochem J 464:241249. 10.1042/BJ20140787

Parpura V, Basarsky TA, Liu F, Jeftinija K, Jeftinija S, and Haydon PG. 1994. Glutamatemediated astrocyte-neuron signalling. Nature 369:744-747. 10.1038/369744a0

Paul R, Phukan BC, Justin Thenmozhi A, Manivasagam T, Bhattacharya P, and Borah A. 2018. Melatonin protects against behavioral deficits, dopamine loss and oxidative stress in homocysteine model of Parkinson's disease. Life Sci 192:238-245. 10.1016/j.1fs.2017.11.016

Peng X, Wood CL, Blalock EM, Chen KC, Landfield PW, and Stromberg AJ. 2003. Statistical implications of pooling RNA samples for microarray experiments. BMC Bioinformatics 4:26. 10.1186/1471-2105-4-26

Poirier Y, Antonenkov VD, Glumoff T, and Hiltunen JK. 2006. Peroxisomal beta-oxidation--a metabolic pathway with multiple functions. Biochim Biophys Acta 1763:1413-1426. 10.1016/j.bbamcr.2006.08.034

Rada JA, and Wiechmann AF. 2009. Ocular expression of avian thymic hormone: changes during the recovery from induced myopia. Mol Vis 15:778-792.

Ramakrishnan S, Sulochana KN, Lakshmi S, Selvi R, and Angayarkanni N. 2006. Biochemistry of homocysteine in health and diseases. Indian J Biochem Biophys 43:275-283.

Raviola E, and Wiesel TN. 1978. Effect of dark-rearing on experimental myopia in monkeys. Invest Ophthalmol Vis Sci 17:485-488.

Reidel B, Goldmann T, Giessl A, and Wolfrum U. 2008. The translocation of signaling molecules in dark adapting mammalian rod photoreceptor cells is dependent on the cytoskeleton. Cell Motil Cytoskeleton 65:785-800. 10.1002/cm.20300

Riddell N, and Crewther SG. 2017a. Integrated Comparison of GWAS, Transcriptome, and Proteomics Studies Highlights Similarities in the Biological Basis of Animal and Human Myopia. Invest Ophthalmol Vis Sci 58:660-669. 10.1167/iovs.16-20618

Riddell N, and Crewther SG. 2017b. Novel evidence for complement system activation in chick myopia and hyperopia models: a meta-analysis of transcriptome datasets. Sci Rep 7:9719. 10.1038/s41598-017-10277-2

Riddell N, Faou P, Murphy M, Giummarra L, Downs RA, Rajapaksha H, and Crewther SG. 2017. The retina/RPE proteome in chick myopia and hyperopia models: Commonalities with inherited and age-related ocular pathologies. Mol Vis 23:872-888.

Riddell N, Giummarra L, Hall NE, and Crewther SG. 2016. Bidirectional Expression of Metabolic, Structural, and Immune Pathways in Early Myopia and Hyperopia. Front Neurosci 10:390. 10.3389/fnins.2016.00390

Rue P, and Martinez Arias A. 2015. Cell dynamics and gene expression control in tissue homeostasis and development. Mol Syst Biol 11:792. 10.15252/msb.20145549 
904

905

906

907

908

909

910

911

912

913

914

915

916

917

918

919

920

921

922

923

924

925

926

927

928

929

930

931

932

933

934

935

936

937

938

939

940

941

942

943

944

945

946

Saw SM, Nieto FJ, Katz J, Schein OD, Levy B, and Chew SJ. 2000. Factors related to the progression of myopia in Singaporean children. Optom Vis Sci 77:549-554.

Schippert R, Schaeffel F, and Feldkaemper MP. 2008. Microarray analysis of retinal gene expression in chicks during imposed myopic defocus. Mol Vis 14:1589-1599.

Schippert R, Schaeffel F, and Feldkaemper MP. 2009. Microarray analysis of retinal gene expression in Egr-1 knockout mice. Mol Vis 15:2720-2739.

Schneider J, Leeder SR, Gopinath B, Wang JJ, and Mitchell P. 2010. Frequency, Course, and Impact of Correctable Visual Impairment (Uncorrected Refractive Error). Survey of Ophthalmology. 10.1016/j.survophthal.2010.02.004

Seet B, Wong TY, Tan DT, Saw SM, Balakrishnan V, Lee LK, and Lim AS. 2001. Myopia in Singapore: taking a public health approach. Br J Ophthalmol 85:521-526.

Sena LA, and Chandel NS. 2012. Physiological roles of mitochondrial reactive oxygen species. Mol Cell 48:158-167. 10.1016/j.molcel.2012.09.025

Shelton L, Troilo D, Lerner MR, Gusev Y, Brackett DJ, and Rada JS. 2008. Microarray analysis of choroid/RPE gene expression in marmoset eyes undergoing changes in ocular growth and refraction. Mol Vis 14:1465-1479.

Shen M, Wang L, Wang B, Wang T, Yang G, Shen L, Wang T, Guo X, Liu Y, Xia Y, Jia L, and Wang X. 2014. Activation of volume-sensitive outwardly rectifying chloride channel by ROS contributes to ER stress and cardiac contractile dysfunction: involvement of CHOP through Wnt. Cell Death Dis 5:e1528. 10.1038/cddis.2014.479

Shih YF, Fitzgerald ME, Norton TT, Gamlin PD, Hodos W, and Reiner A. 1993a. Reduction in choroidal blood flow occurs in chicks wearing goggles that induce eye growth toward myopia. Curr Eye Res 12:219-227.

Shih YF, Fitzgerald ME, and Reiner A. 1993b. Choroidal blood flow is reduced in chicks with ocular enlargement induced by corneal incisions. Current Eye Research 12:229-237.

Shih YF, Fitzgerald ME, and Reiner A. 1993c. Effect of choroidal and ciliary nerve transection on choroidal blood flow, retinal health, and ocular enlargement. Vis Neurosci 10:969979.

Staels B, and Fonseca VA. 2009. Bile acids and metabolic regulation: mechanisms and clinical responses to bile acid sequestration. Diabetes Care 32 Suppl 2:S237-245. 10.2337/dc09S355

Stejskalova L, Vecerova L, Perez LM, Vrzal R, Dvorak Z, Nachtigal P, and Pavek P. 2011. Aryl hydrocarbon receptor and aryl hydrocarbon nuclear translocator expression in human and rat placentas and transcription activity in human trophoblast cultures. Toxicol Sci 123:2636. $10.1093 /$ toxsci/kfr 150

Stone RA, and Khurana TS. 2010. Gene profiling in experimental models of eye growth: clues to myopia pathogenesis. Vision Res 50:2322-2333. 10.1016/j.visres.2010.03.021

Stone RA, McGlinn AM, Baldwin DA, Tobias JW, Iuvone PM, and Khurana TS. 2011. Image defocus and altered retinal gene expression in chick: clues to the pathogenesis of ametropia. Invest Ophthalmol Vis Sci 52:5765-5777. 10.1167/iovs.10-6727

Subramanian A, Tamayo P, Mootha VK, Mukherjee S, Ebert BL, Gillette MA, Paulovich A, Pomeroy SL, Golub TR, Lander ES, and Mesirov JP. 2005. Gene set enrichment analysis: a knowledge-based approach for interpreting genome-wide expression profiles. 
Proceedings of the National Academy of Sciences of the United States of America 102:15545-15550. 10.1073/pnas.0506580102

Tkatchenko AV, Walsh PA, Tkatchenko TV, Gustincich S, and Raviola E. 2006. Form deprivation modulates retinal neurogenesis in primate experimental myopia. Proceedings of the National Academy of Sciences of the United States of America 103:4681-4686. 10.1073/pnas.0600589103

Tripathi S, Glazko GV, and Emmert-Streib F. 2013. Ensuring the statistical soundness of competitive gene set approaches: gene filtering and genome-scale coverage are essential. Nucleic Acids Res 41:e82. 10.1093/nar/gkt054

Veltmann M, Hollborn M, Reichenbach A, Wiedemann P, Kohen L, and Bringmann A. 2016. Osmotic Induction of Angiogenic Growth Factor Expression in Human Retinal Pigment Epithelial Cells. PLoS One 11:e0147312. 10.1371/journal.pone.0147312

Verhoeven VJ, Hysi PG, Wojciechowski R, Fan Q, Guggenheim JA, Hohn R, MacGregor S, Hewitt AW, Nag A, Cheng CY, Yonova-Doing E, Zhou X, Ikram MK, Buitendijk GH, McMahon G, Kemp JP, Pourcain BS, Simpson CL, Makela KM, Lehtimaki T, Kahonen M, Paterson AD, Hosseini SM, Wong HS, Xu L, Jonas JB, Parssinen O, Wedenoja J, Yip SP, Ho DW, Pang CP, Chen LJ, Burdon KP, Craig JE, Klein BE, Klein R, Haller T, Metspalu A, Khor CC, Tai ES, Aung T, Vithana E, Tay WT, Barathi VA, Consortium for Refractive E, Myopia, Chen P, Li R, Liao J, Zheng Y, Ong RT, Doring A, Diabetes C, Complications Trial/Epidemiology of Diabetes I, Complications Research G, Evans DM, Timpson NJ, Verkerk AJ, Meitinger T, Raitakari O, Hawthorne F, Spector TD, Karssen LC, Pirastu M, Murgia F, Ang W, Wellcome Trust Case Control C, Mishra A, Montgomery GW, Pennell CE, Cumberland PM, Cotlarciuc I, Mitchell P, Wang JJ, Schache M, Janmahasatian S, Igo RP, Jr., Lass JH, Chew E, Iyengar SK, Fuchs' Genetics Multi-Center Study G, Gorgels TG, Rudan I, Hayward C, Wright AF, Polasek O, Vatavuk Z, Wilson JF, Fleck B, Zeller T, Mirshahi A, Muller C, Uitterlinden AG, Rivadeneira F, Vingerling JR, Hofman A, Oostra BA, Amin N, Bergen AA, Teo YY, Rahi JS, Vitart V, Williams C, Baird PN, Wong TY, Oexle K, Pfeiffer N, Mackey DA, Young TL, van Duijn CM, Saw SM, Bailey-Wilson JE, Stambolian D, Klaver CC, and Hammond CJ. 2013. Genome-wide meta-analyses of multiancestry cohorts identify multiple new susceptibility loci for refractive error and myopia. Nat Genet 45:314-318. 10.1038/ng.2554

Wallman J, Turkel J, and Trachtman J. 1978. Extreme myopia produced by modest change in early visual experience. Science 201:1249-1251.

Westbrook AM, Crewther DP, and Crewther SG. 1999. Cone receptor sensitivity is altered in form deprivation myopia in the chicken. Optometry and Vision Science 76:326-338.

Wilson C, and Gonzalez-Billault C. 2015. Regulation of cytoskeletal dynamics by redox signaling and oxidative stress: implications for neuronal development and trafficking. Front Cell Neurosci 9:381. 10.3389/fncel.2015.00381

Yang F, He Y, Liu HX, Tsuei J, Jiang X, Yang L, Wang ZT, and Wan YJ. 2014. All-trans retinoic acid regulates hepatic bile acid homeostasis. Biochem Pharmacol 91:483-489. 10.1016/j.bcp.2014.08.018

Yang J, Reinach PS, Zhang S, Pan M, Sun W, Liu B, Li F, Li X, Zhao A, Chen T, Jia W, Qu J, and Zhou X. 2017. Changes in retinal metabolic profiles associated with form deprivation myopia development in guinea pigs. Sci Rep 7:2777. 10.1038/s41598-017-03075-3 
992

993

994

995

996

997

998

999

1000

1001

1002

1003

1004

1005

1006

1007

1008

1009

1010

1011

1012

1013

1014

1015

1016

1017

1018

1019

1020

1021

1022

Yang YS, and Koh JW. 2015. Choroidal Blood Flow Change in Eyes with High Myopia. Korean J Ophthalmol 29:309-314. 10.3341/kjo.2015.29.5.309

Yap M, Cho J, and Woo G. 1990. A survey of low vision patients in Hong Kong. Clinical \& Experimental Optometry 73:19-22.

Yap S, and Naughten E. 1998. Homocystinuria due to cystathionine beta-synthase deficiency in Ireland: 25 years' experience of a newborn screened and treated population with reference to clinical outcome and biochemical control. J Inherit Metab Dis 21:738-747.

Yu H, Ozdemir SS, Koilkonda RD, Chou TH, Porciatti V, Chiodo V, Boye SL, Hauswirth WW, Lewin AS, and Guy J. 2012. Mutant NADH dehydrogenase subunit 4 gene delivery to mitochondria by targeting sequence-modified adeno-associated virus induces visual loss and optic atrophy in mice. Mol Vis 18:1668-1683.

Zhang H, Wong CL, Shan SW, Li KK, Cheng AK, Lee KL, Ge J, To CH, and Do CW. 2011. Characterisation of $\mathrm{Cl}(-)$ transporter and channels in experimentally induced myopic chick eyes. Clin Exp Optom 94:528-535. 10.1111/j.1444-0938.2011.00611.x

Zhang W, Carriquiry A, Nettleton D, and Dekkers JC. 2007. Pooling mRNA in microarray experiments and its effect on power. Bioinformatics 23:1217-1224. 10.1093/bioinformatics/btm081

Zhang Y, Liu Y, Hang A, Phan E, and Wildsoet CF. 2016. Differential gene expression of BMP2 and BMP receptors in chick retina \& choroid induced by imposed optical defocus. Vis Neurosci 33:E015. 10.1017/S0952523816000122

Zhang Y, Liu Y, and Wildsoet CF. 2012. Bidirectional, optical sign-dependent regulation of BMP2 gene expression in chick retinal pigment epithelium. Invest Ophthalmol Vis Sci 53:6072-6080. 10.1167/iovs.12-9917

Zhang Y, and Wildsoet CF. 2015. RPE and Choroid Mechanisms Underlying Ocular Growth and Myopia. Prog Mol Biol Transl Sci 134:221-240. 10.1016/bs.pmbts.2015.06.014

Zhou X, Pardue MT, Iuvone PM, and Qu J. 2017. Dopamine signaling and myopia development: What are the key challenges. Prog Retin Eye Res 61:60-71. 10.1016/j.preteyeres.2017.06.003

Zwart SR, Gibson CR, Mader TH, Ericson K, Ploutz-Snyder R, Heer M, and Smith SM. 2012. Vision changes after spaceflight are related to alterations in folate- and vitamin B-12dependent one-carbon metabolism. J Nutr 142:427-431. 10.3945/jn.111.154245 


\section{Table $\mathbf{1}$ (on next page)}

Pathways enriched between $6 \mathrm{~h}$ and $72 \mathrm{~h}$ of FD induction

Mean Normalised Enrichment Score (NES) and false discovery rate (FDR) for the biological pathways identified by GSEA in FD induction. Most genes responding to FD are involved in mitochondrial energy metabolism. The NES reflects the degree to which a set of genes is over-represented at either the top or bottom of a ranked list of genes while also taking into account differences in pathway size (i.e. gene-set size) and is the primary statistic for examining enrichment results, and for comparing results across pathways. 


\begin{tabular}{|c|c|c|c|c|}
\hline Pathway & Core Genes & Database & NES & FDR \\
\hline Huntingtons Disease & $\begin{array}{l}\text { APAF1, ATP5C1, ATP5D, ATP5E, ATP5F1, ATP5H, ATP5O, BDNF, } \\
\text { CASP3, CASP8, CLTA, COX4I1, COX5A, COX6A1, COX6C, COX7A2, } \\
\text { COX7A2L, COX7C, COX8A, CYCS, DNAH3, DNAI1, DNAL1, DNALI1, } \\
\text { GPX1, HDAC2, IFT57, NDUFA1, NDUFA10, NDUFA2, NDUFA4, } \\
\text { NDUFA5, NDUFA6, NDUFA7, NDUFA8, NDUFB1, NDUFB10, NDUFB3, } \\
\text { NDUFB4, NDUFB5, NDUFB6, NDUFB8, NDUFB9, NDUFC1, NDUFC2, } \\
\text { NDUFS1, NDUFS3, NDUFS4, NDUFS6, NDUFS7, NDUFS8, NDUFV3, } \\
\text { PLCB1, PLCB4, POLR2D, POLR2F, POLR2J, POLR2L, PPID, SDHA, } \\
\text { SLC25A4, SLC25A6, SOD2, TAF4, TBP, TBPL1, TFAM, UQCR10, } \\
\text { UQCR11, UQCRFS1, UQCRH, VDAC3 }\end{array}$ & KEGG & 1.76 & 0.15 \\
\hline Oxidative Phosphorylation & $\begin{array}{l}\text { ATP5C1, ATP5D, ATP5E, ATP5F1, ATP5H, ATP5I, ATP5J2, ATP5O, } \\
\text { ATP6V0D2, ATP6V1G1, COX11, COX15, COX17, COX411, COX5A, } \\
\text { COX6A1, COX6C, COX7A2, COX7A2L, COX7C, COX8A, NDUFA1, } \\
\text { NDUFA10, NDUFA2, NDUFA4, NDUFA5, NDUFA6, NDUFA7, NDUFA8, } \\
\text { NDUFB1, NDUFB10, NDUFB3, NDUFB4, NDUFB5, NDUFB6, NDUFB8, } \\
\text { NDUFB9, NDUFC1, NDUFC2, NDUFS1, NDUFS3, NDUFS4, NDUFS6, } \\
\text { NDUFS7, NDUFS8, NDUFV3, PPA1, PPA2, SDHA, UQCR10, UQCR11, } \\
\text { UQCRFS1, UQCRH }\end{array}$ & KEGG & 1.81 & 0.16 \\
\hline Mitochondrial Protein Import & $\begin{array}{l}\text { BCS1L, CHCHD4, COX17, DNAJC19, GRPEL1, HSCB, HSPD1, PAM16, } \\
\text { PMPCA, PMPCB, SAMM50, SLC25A12, SLC25A4, SLC25A6, TIMM13, } \\
\text { TIMM17A, TIMM22, TIMM44, TIMM9, TOMM22, TOMM5, TOMM7 }\end{array}$ & Reactome & 1.80 & 0.16 \\
\hline One Carbon Pool By Folate & ATIC, DHFR, GART, MTFMT, MTHFD1, MTHFD1L, MTHFS, TYMS & KEGG & 1.74 & 0.17 \\
\hline Cholesterol Biosynthesis & $\begin{array}{l}\text { DHCR7, FDFT1, GGPS1, HMGCR, HMGCS1, IDI1, MSMO1, NSDHL, } \\
\text { SQLE }\end{array}$ & Reactome & 1.77 & 0.17 \\
\hline Antigen Processing Cross Presentation & $\begin{array}{l}\text { CD36, CTSS, NCF4, PSMA1, PSMA2, PSMA3, PSMA5, PSMA6, PSMA7, } \\
\text { PSMB1, PSMB2, PSMB3, PSMC1, PSMC2, PSMC3, PSMC5, PSMD1, } \\
\text { PSMD10, PSMD3, PSMD5, RPS27A, SEC61B, SEC61G, TAP1, UBA52 }\end{array}$ & Reactome & 1.74 & 0.18 \\
\hline Alzheimers Disease & $\begin{array}{l}\text { NDUFB6, CYCS, NDUFB3, NDUFA8, UQCR11, NDUFC2, ATP5F1, } \\
\text { ATP5E, NDUFB5, UQCR10, COX4I1, COX6A1, COX7C, NDUFB10, } \\
\text { NDUFB1, CASP8, NDUFV3, NDUFA6, ATP5H, COX7A2, RYR3, } \\
\text { NDUFA2, NDUFC1, NDUFS1, NDUFA1, SDHA, NDUFB8, NDUFA4, } \\
\text { NDUFA5, APAF1, UQCRH, ATP5O, NDUFA7, NDUFS6, NDUFS4, } \\
\text { ATP5D, ATP5C1, NDUFB9, CACNA1D, NDUFS8, NDUFS3, NDUFS7, } \\
\text { CASP3, COX8A, COX6C, COX5A, NDUFA10, ATP2A1, COX7A2L, } \\
\text { TNFRSF1A, UQCRFS1, PSEN1, NDUFB4, IL1B, PLCB1, NCSTN, PLCB4 }\end{array}$ & KEGG & 1.74 & 0.19 \\
\hline Parkinsons Disease & $\begin{array}{l}\text { APAF1, ATP5C1, ATP5D, ATP5E, ATP5F1, ATP5H, ATP5O, CASP3, } \\
\text { COX411, COX5A, COX6A1, COX6C, COX7A2, COX7A2L, COX7C, } \\
\text { COX8A, CYCS, GPR37, NDUFA1, NDUFA10, NDUFA2, NDUFA4, } \\
\text { NDUFA5, NDUFA6, NDUFA7, NDUFA8, NDUFB1, NDUFB10, NDUFB3, }\end{array}$ & KEGG & 1.77 & 0.19 \\
\hline
\end{tabular}


TCA Cycle And Respiratory Electron Transport

Respiratory Electron Transport

Respiratory Electron Transport ATP Synthesis By Chemiosmotic Coupling And Heat Production By Uncoupling Proteins

Translation

ER Phagosome Pathway
NDUFB5, NDUFB6, NDUFB8, NDUFB9, NDUFC1, NDUFC2, NDUFS1, NDUFS3, NDUFS4, NDUFS6, NDUFS7, NDUFS8, NDUFV3, PARK7, PPID, SDHA, SLC25A4, SNCAIP, UBE2L3, UQCR10, UQCR11, UQCRH, VDAC3

ATP5C1, ATP5D, ATP5E, ATP5F1, ATP5H, ATP5I, ATP5J2, ATP5O,

COX4I1, COX5A, COX6A1, COX6C, COX7A2L, COX7C, COX8A, CYCS, D2HGDH, DLD, IDH3A, LDHB, NDUFA1, NDUFA10, NDUFA2,

NDUFA4, NDUFA5, NDUFA6, NDUFA7, NDUFA8, NDUFB1, NDUFB10, NDUFB3, NDUFB5, NDUFB6, NDUFB8, NDUFB9, NDUFC1, NDUFC2, NDUFS1, NDUFS3, NDUFS4, NDUFS6, NDUFS7, NDUFS8, NDUFV3,

NNT, SDHA, SUCLG1, SUCLG2, UQCR11, UQCRH

COX4I1, COX5A, COX6A1, COX6C, COX7A2L, COX7C, COX8A, CYCS, Reactome

NDUFA1, NDUFA10, NDUFA12, NDUFA2, NDUFA4, NDUFA5,

NDUFA6, NDUFA7, NDUFA8, NDUFB1, NDUFB10, NDUFB3, NDUFB4,

NDUFB5, NDUFB6, NDUFB8, NDUFB9, NDUFC1, NDUFC2, NDUFS1,

NDUFS3, NDUFS4, NDUFS6, NDUFS7, NDUFS8, NDUFV3, SDHA,

UQCR11, UQCRFS1, UQCRH

ATP5C1, ATP5D, ATP5E, ATP5F1, ATP5H, ATP5I, ATP5J2, ATP5O,

COX4I1, COX5A, COX6A1, COX6C, COX7A2L, COX7C, COX8A, CYCS,

Reactome

NDUFA1, NDUFA10, NDUFA12, NDUFA2, NDUFA4, NDUFA5,

NDUFA6, NDUFA7, NDUFA8, NDUFB1, NDUFB10, NDUFB3, NDUFB4,

NDUFB5, NDUFB6, NDUFB8, NDUFB9, NDUFC1, NDUFC2, NDUFS1,

NDUFS3, NDUFS4, NDUFS6, NDUFS7, NDUFS8, NDUFV3, SDHA,

UCP3, UQCR11, UQCRFS1, UQCRH

EEF1B2, EIF2B1, EIF2B2, EIF2S1, EIF2S2, EIF2S3, EIF3D, EIF3H, EIF3I, EIF3J, EIF4EBP1, EIF4H, EIF5B, RPL10A, RPL11, RPL13, RPL14, RPL18A, RPL19, RPL21, RPL22, RPL23A, RPL24, RPL26L1, RPL27, RPL27A, RPL29, RPL30, RPL32, RPL35, RPL35A, RPL36, RPL36A, RPL37, RPL37A, RPL38, RPL39, RPL5, RPL6, RPL7, RPL8, RPLP1, RPLP2, RPN1, RPS10, RPS11, RPS14, RPS15, RPS15A, RPS16, RPS2, RPS20, RPS23, RPS24, RPS25, RPS26, RPS27A, RPS28, RPS29, RPS3, RPS3A, RPS4X, RPS6, RPS7, RPS8, RPSA, SEC61B, SEC61G, SPCS1, SPCS2, SPCS3, SRP19, SRP72, SSR3, UBA52

PSMA1, PSMA2, PSMA3, PSMA5, PSMA6, PSMA7, PSMB1, PSMB2, PSMB3, PSMC1, PSMC2, PSMC3, PSMC5, PSMD1, PSMD10, PSMD3, 


\section{Table 2 (on next page)}

Summary statistics for clusters of pathways enriched during FDMI and normal development

Mean Normalised Enrichment Score (NES), false discovery rate (FDR) and signal strength statistic (Signal) for the biological pathways implicated by GSEA in control and FDM. Normal eye development implicated 10 cluster of pathways showing average signal strength while form-deprivation induction implicated 18 clusters of pathways. Pathways shown here only include clustered pathways as represented in Fig. 3 and 4 and do not include pathways that were unclustered. Further detail on the unclustered pathways can be found in Supplementary File 1 . The NES reflects the degree to which a set of genes is overrepresented at either the top or bottom of a ranked list of genes while also taking into account differences in pathway size (i.e. geneset size). NES is the primary statistic for examining enrichment results, and for comparing results across pathways. The percentage signal strength statistic reflects the proportion of the core set of genes that contribute most to a given pathway's enrichment) by accounting for particular genes position in the ranked list. A high signal strength indicates that the genes within a pathway are located close to the top (positive NES) or bottom (negative NES) of the ranked gene list. If the core genes are spread throughout the ranked list, then the signal strength decreases towards zero (Mootha, Lindgren et al. 2003, Subramanian, Tamayo et al. 2005). 


\begin{tabular}{|c|c|c|c|c|c|c|c|c|}
\hline \multirow[b]{3}{*}{ Cluster } & & & & & & & & \\
\hline & \multicolumn{4}{|c|}{ Control } & \multicolumn{4}{|c|}{ FDMI } \\
\hline & Pathways in Cluster & NES & FDR q-value & Signal & Pathways in Cluster & NES & FDR q-value & Signal \\
\hline Cell cycle, mitotic & 5 & -2.233 & 0.019 & $31 \%$ & 3 & -2.253 & 0.009 & $98 \%$ \\
\hline Cell maintenance \& survival & - & - & - & - & 28 & -2.180 & 0.014 & $117 \%$ \\
\hline Clatherin-mediated endocytosis (CME) & - & - & - & - & 2 & -2.014 & 0.032 & $81 \%$ \\
\hline Complement and coagulation cascades (CCC) & - & - & - & - & 2 & 2.708 & 0.003 & $140 \%$ \\
\hline Cytochrome p450 & 2 & 2.247 & 0.025 & $47 \%$ & 5 & 2.629 & 0.002 & $133 \%$ \\
\hline Cytokine pathways & 2 & 2.717 & 0.002 & $40 \%$ & - & - & - & - \\
\hline Neuron structure/growth & - & - & - & - & 5 & -2.321 & 0.007 & $80 \%$ \\
\hline Fatty acid (FA) metabolism & - & - & - & - & 2 & -1.886 & 0.032 & $84 \%$ \\
\hline Glucosaminoglycan (GAG) Metabolism & 4 & 2.121 & 0.029 & $40 \%$ & - & - & - & - \\
\hline Ion channel transport & - & - & - & - & 2 & -2.331 & 0.007 & $99 \%$ \\
\hline Mitochondrial energy metabolism & - & - & - & - & 7 & 2.396 & 0.005 & $47 \%$ \\
\hline Neurotransmission & 5 & -2.522 & 0.011 & $37 \%$ & 8 & -2.423 & 0.007 & $103 \%$ \\
\hline Peroxisome & 2 & -2.221 & 0.017 & $40 \%$ & - & - & - & - \\
\hline Phospholipid metabolism & 2 & -2.134 & 0.026 & $28 \%$ & 3 & -1.939 & 0.027 & $57 \%$ \\
\hline Signal transduction, growth factors (GF) & - & - & - & - & 4 & -1.984 & 0.021 & $66 \%$ \\
\hline \multicolumn{9}{|l|}{ Signal transduction, g-protein coupled } \\
\hline receptors (GPCR) & 7 & 2.572 & 0.019 & $52 \%$ & 7 & 2.683 & 0.010 & $122 \%$ \\
\hline \multicolumn{9}{|l|}{ Signal transduction, mitogen-activated protein } \\
\hline kinases (MAPK) & - & & - & - & 4 & -1.931 & 0.028 & $67 \%$ \\
\hline Signal transduction, nerve growth factor (NGF) & - & - & - & - & 5 & -2.195 & 0.014 & $84 \%$ \\
\hline
\end{tabular}




\begin{tabular}{|c|c|c|c|c|c|c|c|c|}
\hline Transcription & - & - & - & - & 10 & -2.218 & 0.013 & $120 \%$ \\
\hline Translation & - & - & - & - & 8 & 2.731 & 0.001 & $46 \%$ \\
\hline Ubiquitin-mediated proteolysis & 3 & -2.491 & 0.006 & $39 \%$ & - & - & - & - \\
\hline Vesicle-mediated transport & 3 & -2.152 & 0.025 & $40 \%$ & 3 & -2.097 & 0.013 & $103 \%$ \\
\hline
\end{tabular}




\section{Figure 1}

MRI images of chick eye following 10 days of FDM induction and 3 days recovery

(A) Monocular form-deprivation of the right eye (RE) for 10 days demonstrates abnormal ocular growth, excess vitreal volume, and thinned choroid of RE compared to its fellow left eye (LE). (B) 72 hours post-occlusion recovery (ie. 72 hours normal visual experience) resulted in vitreous volume decrease and choroidal expansion. Previous studies (Liang, Crewther et al. 2004) have shown 300\% in RE compared to fellow LE choroidal thickness 3 days later. Note: Images (same magnification) in (B) slightly more dorsal than in (A). Image credit: G. Eagan
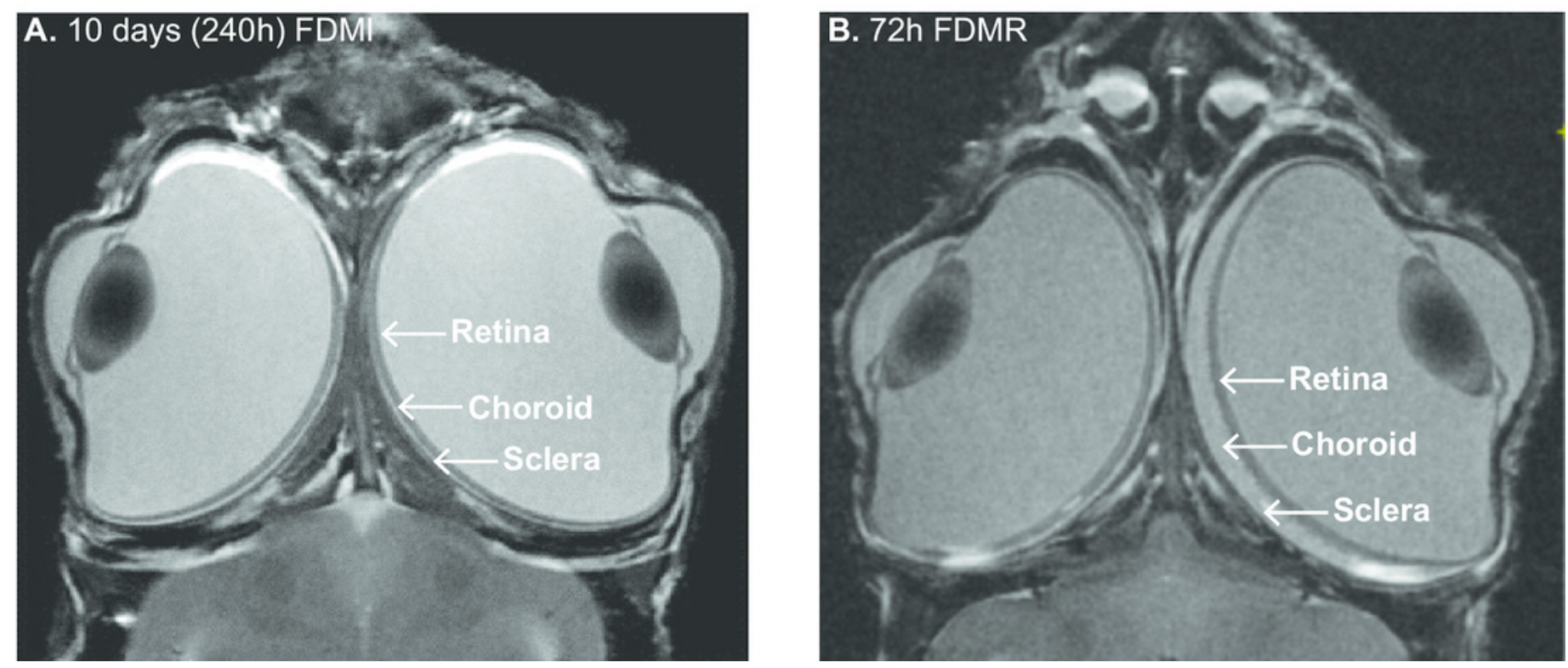


\section{Figure 2}

Ocular Biometrics for FDMI and FDMR

Mean $( \pm S E)$ measures of refractive status, axial length $(A L)$ and vitreous chamber depth (VCD). To complement McGlinn et al (2007), refraction (A) and AL \& VCD (B) were collected during $6 \mathrm{~h}$ and $72 \mathrm{~h}$ of normal development and $6 \mathrm{~h}$ and $72 \mathrm{~h}$ following 7 -days induction of myopia. Refraction, AL \& VCD measures for $24 \mathrm{~h}$ recovery after prolonged form deprivation is shown in (C) and (D). Both refractive state and axial length changes were highly correlated $(r=.78)$ during occluder wear (E). Note: Measures for anterior chamber and lens thickness are included in Supplementary Figure 1. 
a

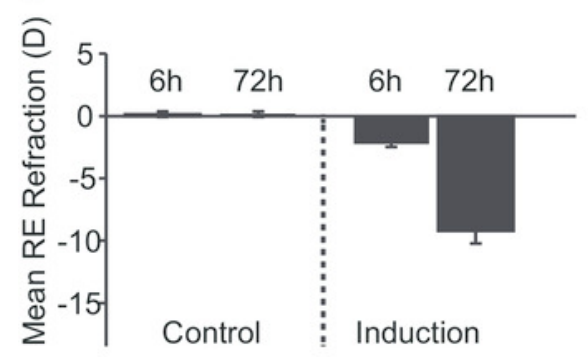

b

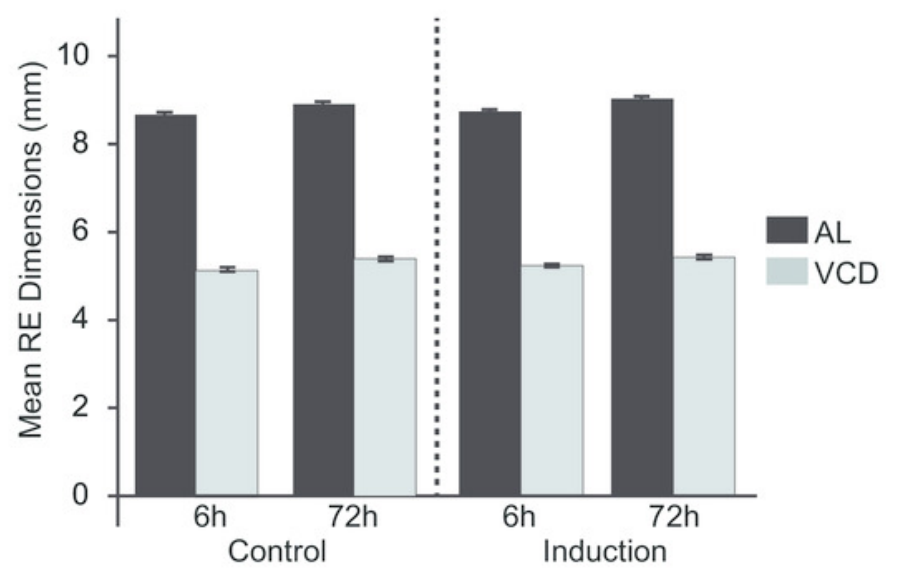

d

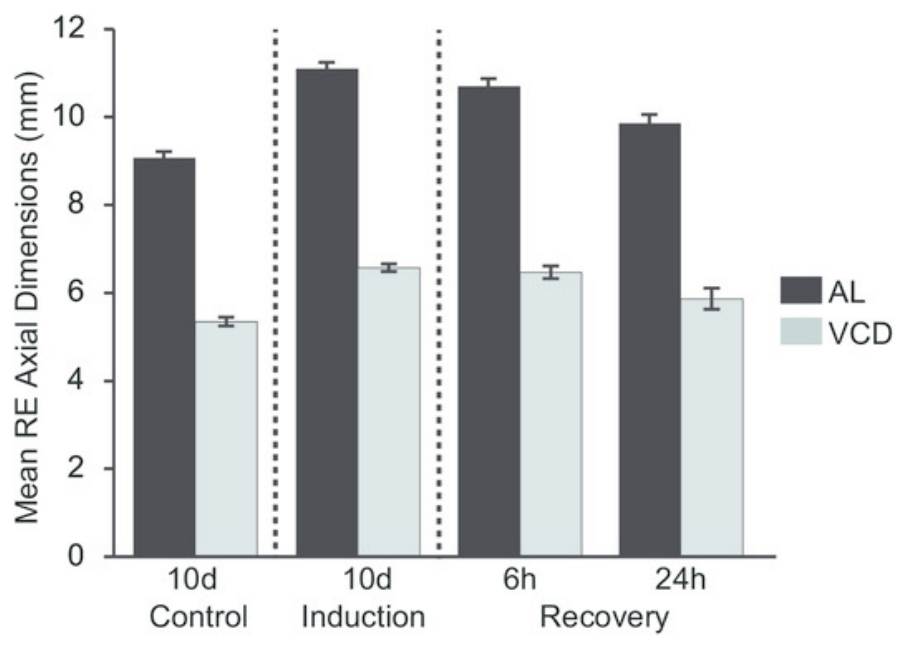

e

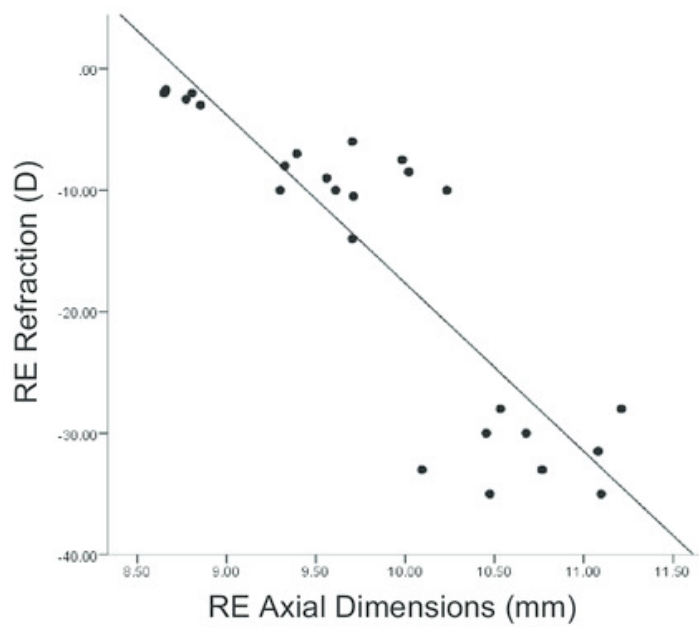




\section{Figure 3 (on next page)}

Enrichment map for highly clustered pathways in normal eye development

Gene set enrichment analysis revealed 61 biological pathways that can be functionally grouped into 10 clusters using a co-efficient of similarity) altered during the 10 days of normal eye development in retina/RPE/choroid. Note: Each node represents a biological pathway from supplementary File 1 . The colour of each node emphasises the direction of expression and normalised enrichment score (NES). Node size is relative to the number of genes in the pathway. Thickness of the connections (green) between each node reflects the degree of similarity between each gene set. Twenty-six pathways did not meet the clustering similarity coefficient of 0.5 and hence are not shown here. Note cluster names: GAG, glycosaminoglycan; GPCR, g-protein coupled receptors 


\section{Normal Development}

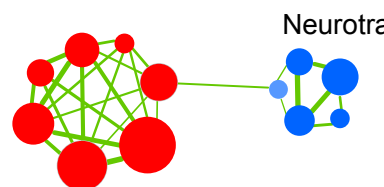

Cell cycle, mitotic

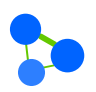

Ubiquitin-mediated proteolysis
Vesicle-mediated transport

Signal transduction, GPCR

of GAG metabolism $\left\{\begin{array}{l}\text { Cytokine } \\ \text { pathways }\end{array} \int_{\text {p450 }}^{\text {Cytochrome }} \begin{array}{l}\text { Phospholipid } \\ \text { metabolism }\end{array}\right\}$ Peroxisome

Not clustered

Figure Key:

Expression

increase

Expression

decrease

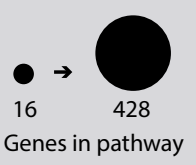




\section{Figure 4 (on next page)}

Enrichment map for highly clustered pathways in form deprivation induction and recovery

Axial elongation during 10 days of form-deprivation compared to normal unoccluded controls resulted in 130 altered pathways in retina/RPE choroid (inner node) while 24h recovery (outer annulus) identified only 1 statistically significant pathway i.e Bile acid \& bile salt metabolism. Pathways not statistically enriched during FDMR are shown for comparison purposes. Notably, expression profiles of FDMI and FDMR are consistent despite the fact that only the FDMR data includes choroidal tissue. Pathways highly expressed during induction (red inner node) were often suppressed during normal vision and recovery (blue outer annulus) and vice versa. Note: Each node represents a biological pathway from supplementary File 1. The colour of each node emphasises the direction of expression and normalised enrichment score (NES). Node size is relative to the number of genes in the pathway. Thickness of the connections (green) between each node reflects the degree of similarity between each gene set. There were 22 unclustered pathways in FDMI that did not meet the clustering similarity coefficient of 0.5. Note cluster names: CCC, complement and coagulation cascades; CME, clatherin-mediated endocytosis; FA, Fatty acid; GF, growth factors; GPCR, g-protein coupled receptors; MAPK, mitogen-activated protein kinases; NGF, nerve growth factor 


\section{Form deprivation myopia induction}

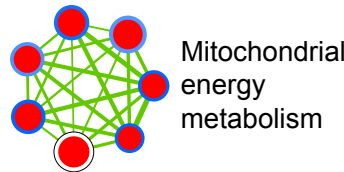

$\int^{0} \operatorname{ccc}$
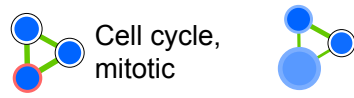

Phospholipid metabolism

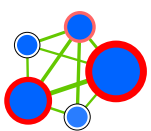

Neuron

structure/growth

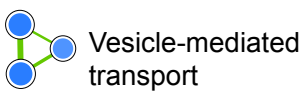

FA

metabolism

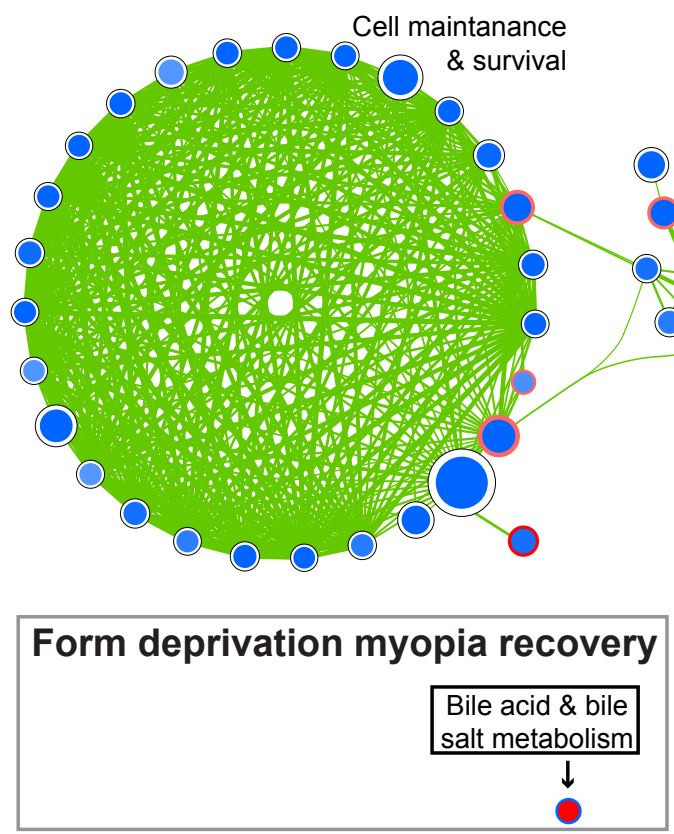

Transcription

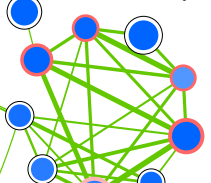

Not clustered 00000000000000000000
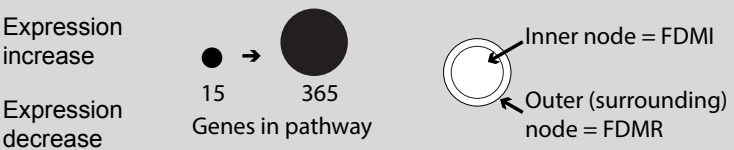
Figure 5 (on next page)

Median expression of pathways involved in mitochondrial metabolism

Graphs of the seven mitochondrial metabolism pathways with significant expression shifts across $240 \mathrm{~h}$ of occluder wear relative to unoccluded controls (A) Alzheimer's disease, (B) Huntington's disease, (C) Parkinson's disease, (D) Oxidative phosphorylation, (E) Respiratory electron transport, (F) Respiratory electron transport/ATP synthesis by chemiosmotic coupling and heat production by uncoupling proteins, (G) TCA cycle and respiratory electron transport. 


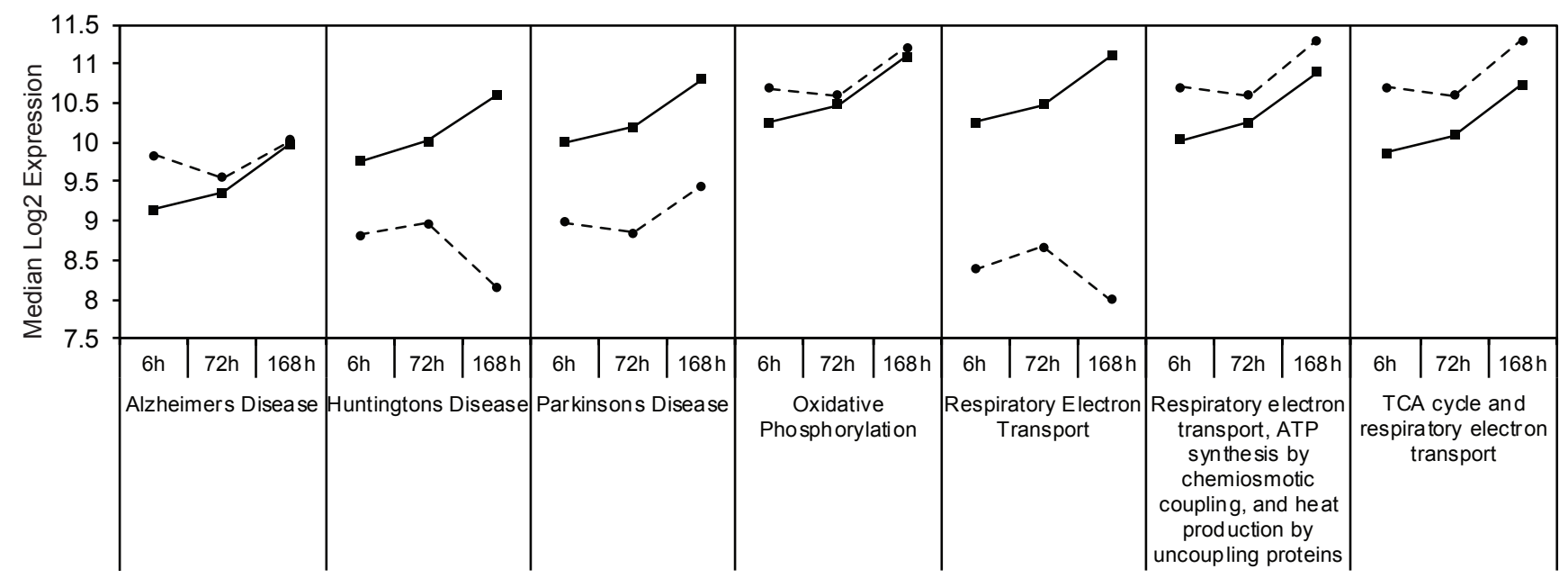




\section{Figure 6 (on next page)}

Median expression of pathways involved in neurotransmission during normal ocular development and in FDMI

Graphs of the neurotransmission-related pathways with significant expression shifts during normal ocular development (dotted lines) and FDMI (solid lines) are shown. (A-D) Four pathways were significant in both normal development and FDMI. The leading-edge subsets for these pathways identified 115 common core genes shared within these pathways during normal development and during FDMI and 27 other core genes specific to normal development and 9 specific to FDMI (Supplementary file 1). (E-H) Graphs indicate FDMI induced down regulation of expression shift in 4 additional neurotransmission-related pathways with significant expression shifts during FDMI (solid lines) only. These pathways were not significant during normal ocular development but data are shown for comparison purposes (dotted lines). (A) Neuronal system (B) Neurotransmitter release cycle (C) Neurotransmitter receptor binding $\&$ downstream transmission in the postsynaptic cell (D) Transmission across chemical synapse (E) Activation of NMDA receptor upon glutamate binding and postsynaptic events (F) CREB phosphorylation through the activation of RAS (G) Long-term potentiation $\mathbf{( H )}$ Post-NMDA receptor activation events 
Neurofransminterer receptor binding \& downstream

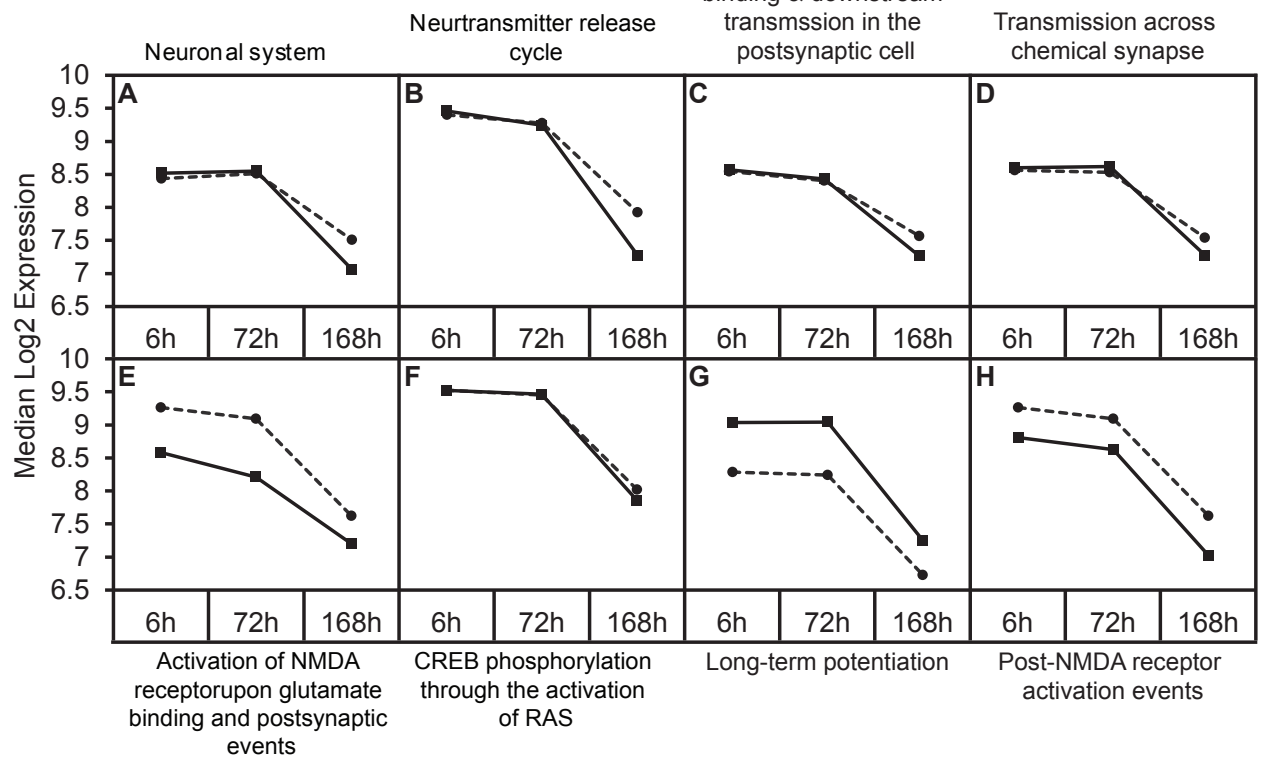


Figure 7 (on next page)

Median expression of pathways involved in ion transport during normal ocular development and in FDMI

Expression of the (A) lon channel transport and (B) Ligand-gated ion channel transport pathways with significant expression shifts during FDMI (solid lines) compared to normal development (dotted lines) are shown. The 'Ligand-gated ion channel transport pathway' pathway was also significantly altered during normal development and was clustered with the neurotransmission pathway. 


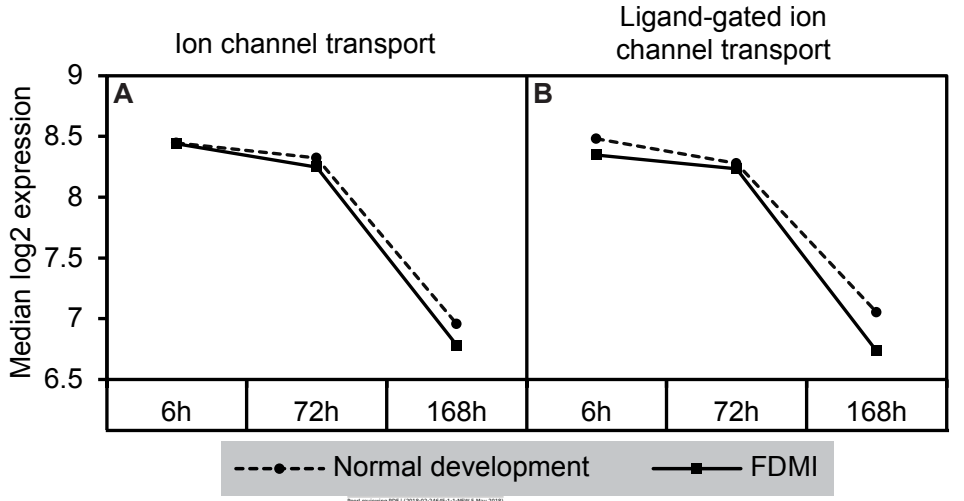


Figure 8 (on next page)

Median expression of pathways involved in the complement and coagulation cascade

Graphs indicate greater expression shift in the complement $\&$ coagulation cascade between $72 \mathrm{~h}$ and $240 \mathrm{~h}$ of occluder wear for both (A) formation of fibrin clot/clotting cascade and (B) complement and coagulation cascades. Note that the 'complement and coagulation cascades' pathway was also significantly altered during normal development. 
Formation of fibrin clot/clotting
Complement and coagulation

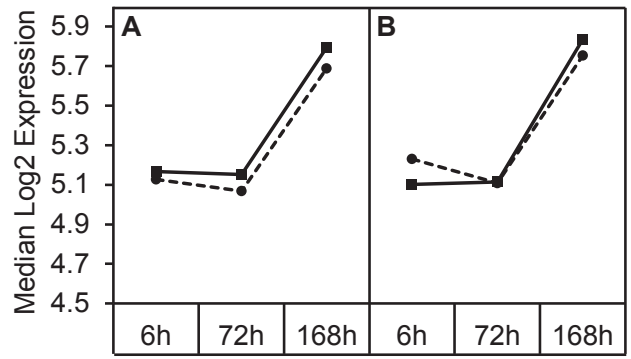

---.-- Normal development 
Figure 9 (on next page)

Median expression of pathways involved in cytochrome p450 metabolism

Graph indicates enhanced expression in cytochrome p450 related pathways in FDMI compared to normal development. (A) Biological oxidations (B) Cytochrome P450 arranged by substrate type (C) Drug metabolism, cytochrome P450 (D) Drug metabolism of xenobiotics by cytochrome P450 (E) Phase 1 functionalization of compounds 


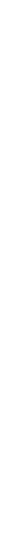


Figure 10 (on next page)

Median expression of core genes in the bile acid and bile salt metabolism pathway during FDM

Graphs shows median change of the core genes during normal ocular development, FDMI, and FDMR. This pathway was found to be significant for FDMI (left, sold line) and FDMR (right, solid line) but not in normal development. Note: Median expression value was calculated based on core genes identified in each experimental group. This pathway was not significantly altered during normal ocular development but data are shown for comparison purposes (dotted line). 


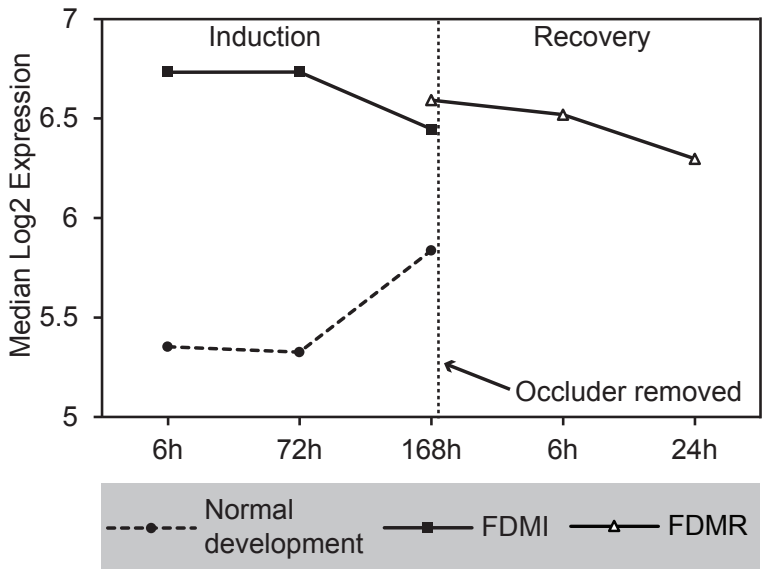

